Supporting Information

\title{
Aerobic Oxidative Sulfenylation of Pyrazolones and Pyrazoles Catalyzed by Metal-Free Flavin-Iodine Catalysis
}

Kazumasa Tanimoto, Ryoma Ohkado, and Hiroki Iida*

Department of Chemistry, Graduate School of Natural Science and Technology,

Shimane University, 1060 Nishikawatsu, Matsue, 690-8504, Japan

E-mail address: iida@riko.shimane-u.ac.jp

1. Results of Catalytic Sulfenylation $\quad$ S2

2. ${ }^{1} \mathrm{H}$ and ${ }^{13} \mathrm{C}\left\{{ }^{1} \mathrm{H}\right\}$ NMR Spectra of Novel Compounds S5

3. ${ }^{1} \mathrm{H}$ and ${ }^{13} \mathrm{C}\left\{{ }^{1} \mathrm{H}\right\}$ NMR Spectra of Known Compounds $\quad$ S9

4. References $\quad$ S25 


\section{Results of Catalytic Sulfenylation}

\section{Effects of Solvents and Iodine Sources}

The reaction condition for the catalytic sulfenylation of $1 \mathbf{a}$ with $\mathbf{2 a}$ was optimized as shown in Table S1.

Table S1. Screening of solvents and iodine sources ${ }^{a}$

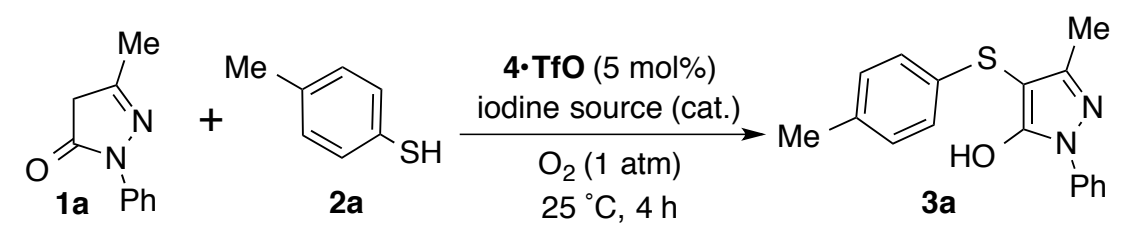

\begin{tabular}{llll}
\hline entry & solvent & iodine & yield of 3a $(\%)^{b}$ \\
\hline 1 & $\mathrm{MeOH}$ & $\mathrm{I}_{2}(5 \mathrm{~mol} \%)$ & 8 \\
2 & $t$-BuOH & $\mathrm{I}_{2}(5 \mathrm{~mol} \%)$ & 15 \\
3 & $\mathrm{CH}_{3} \mathrm{CN}$ & $\mathrm{I}_{2}(5 \mathrm{~mol} \%)$ & 70 \\
4 & $\mathrm{DMF}$ & $\mathrm{I}_{2}(5 \mathrm{~mol} \%)$ & 30 \\
5 & $\mathrm{DCE}$ & $\mathrm{I}_{2}(5 \mathrm{~mol} \%)$ & 31 \\
6 & $\mathrm{CHCl}_{3}$ & $\mathrm{I}_{2}(5 \mathrm{~mol} \%)$ & 19 \\
7 & $\mathrm{AcOEt}$ & $\mathrm{I}_{2}(5 \mathrm{~mol} \%)$ & 27 \\
8 & $\mathrm{CH}_{3} \mathrm{CN}$ & $\mathrm{TBAI}(10 \mathrm{~mol} \%)$ & 32 \\
9 & $\mathrm{CH}_{3} \mathrm{CN}$ & $\mathrm{NH} 4 \mathrm{I}(10 \mathrm{~mol} \%)$ & 32 \\
10 & $\mathrm{CH}_{3} \mathrm{CN}$ & $\mathrm{KI}(10 \mathrm{~mol} \%)$ & 30 \\
11 & $\mathrm{CH}_{3} \mathrm{CN}$ & $\mathrm{HI}(10 \mathrm{~mol} \%)$ & 64 \\
\hline
\end{tabular}

${ }^{a}$ Conditions: 1a $(0.5 \mathrm{M}), 2 \mathrm{a}(0.55 \mathrm{M}), \mathbf{4} \cdot \mathbf{T f O}(5 \mathrm{~mol} \%)$, iodine source (5 or $\left.10 \mathrm{~mol} \%\right)$, and solvent under $\mathrm{O}_{2}(1 \mathrm{~atm})$ at $25{ }^{\circ} \mathrm{C}$ for $4 \mathrm{~h} . \quad{ }^{b}$ Determined by ${ }^{1} \mathrm{H}$ NMR. 


\section{Effects of Catalysts}

To examine the catalytic activity of diverse riboflavin-derived catalysts with varied redox properties for the sulfenylation of 1 a with $\mathbf{2 a}$ (Table S2), we undertook a study to compare the reactivities of related catalysts ${ }^{\mathrm{S} 1}$, which revealed that the catalytic activity observed for the cationic flavins (4•TfO, 11•TfO, and 12•TfO) is higher than that observed with the neutral riboflavin (10a) and riboflavin tetraacetate $(\mathbf{1 0 b})$. Among the three cationic flavins, 5-ethyl alloxazinium 4•TfO was the most efficient catalyst, which was followed by the 5-ethyl isoalloxazinium 12•TfO and 1,10-bridged alloxazinium 11•TfO. Overall, the electropositive flavin catalysts, such as $\mathbf{4} \cdot \mathbf{T f O}$ and 12-TfO, which show positive reduction potentials $\left(E_{1}\right.$ and $\left.E_{2}\right)$ seem to be favorable in this sulfenylation. On the other hand, the catalytic activity of $\mathbf{1 2} \cdot \mathbf{T f O}$, which displayed the most positive reduction potential, was slightly lower than that of $\mathbf{4} \cdot \mathbf{T f O}$. The electrophilic character of 5-ethyl isoalloxazinium salt and 5-ethyl alloxazinium salt are known to be largely different, and therefore cause a change of the rate-limiting step in the flavin-catalyzed oxidation with $\mathrm{H}_{2} \mathrm{O}_{2} .{ }^{\mathrm{S} 2}$ In a similar way, not only the redox potential but also the electrophilicity of the catalyst would influence the catalytic activity of the present aerobic sulfenylation.

Table S2. Comparison with catalytic activity of flavins for aerobic sulfenylation of $\mathbf{1 a}$ with $\mathbf{2} \mathbf{a}^{a}$
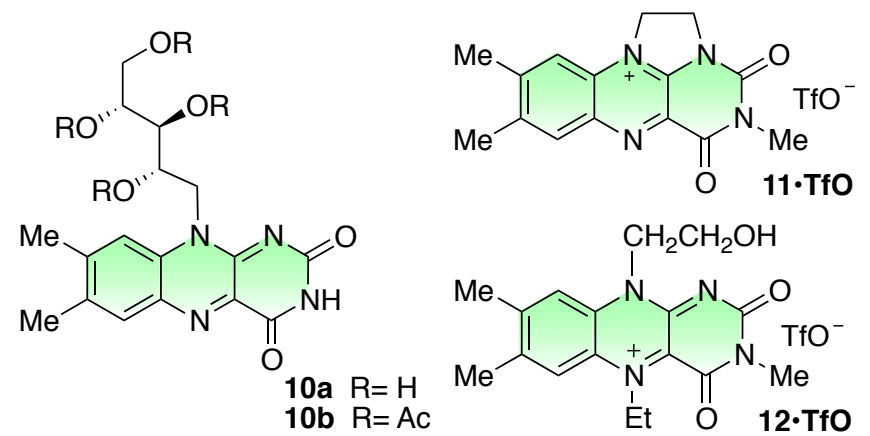

\begin{tabular}{ccccc}
\hline entry & flavin & $E_{1}(\mathrm{~V} \text { vs SCE })^{b}$ & $E_{2}(\mathrm{~V} \text { vs SCE })^{b}$ & yield (\%) \\
\hline 1 & $\mathbf{1 0 a}^{c}$ & - & - & 1 \\
2 & $\mathbf{1 0 b}$ & $-1.18^{d}$ & $-1.87^{d}$ & 3 \\
3 & $\mathbf{1 1 \bullet T f O}$ & -0.255 & -1.25 & 19 \\
4 & $\mathbf{4}$ TfO & -0.026 & -0.787 & 94 \\
5 & $\mathbf{1 2 \cdot T f O}$ & 0.263 & -0.365 & 57 \\
\hline
\end{tabular}

${ }^{a}$ Conditions: 1a $(1.0 \mathrm{M}), 2 \mathrm{a}(1.1 \mathrm{M})$, flavin $(3 \mathrm{~mol} \%), \mathrm{I}_{2}(5 \mathrm{~mol} \%)$, and $\mathrm{CH}_{3} \mathrm{CN}$ under $\mathrm{O}_{2}$, atmosphere $(1 \mathrm{~atm})$ at $25^{\circ} \mathrm{C}$ for $8 \mathrm{~h}$. Yield was determined by ${ }^{1} \mathrm{H}$ NMR using 1,3,5-trioxane as the internal standard. ${ }^{b}$ Reversible redox potentials of the first $\left(E_{1}\right)$ and second reductions $\left(E_{2}\right)$ measured by cyclic voltammetry relative to Standard Calomel Electrode (SCE). From ref 17. ${ }^{c} \mathbf{1 0 a}$ was insoluble in $\mathrm{CH}_{3} \mathrm{CN} .{ }^{d}$ From ref $\mathrm{S} 3$. 


\section{Aerobic Sulfenylation of Electron-Rich Benzenes}

The flavin-iodine-catalyzed reaction of the electron-rich 1,3,5-trimethoxybenzene (8a) furnished the corresponding sulfide (9a) in 91\% yield (Scheme S1A). Sulfenyl aniline (8b) was also effectively converted to the corresponding disulfenyl aniline (9b) in good yield (Scheme S1B).

Scheme S1. Aerobic sulfenylation of aromatic rings bearing electron-donating groups.
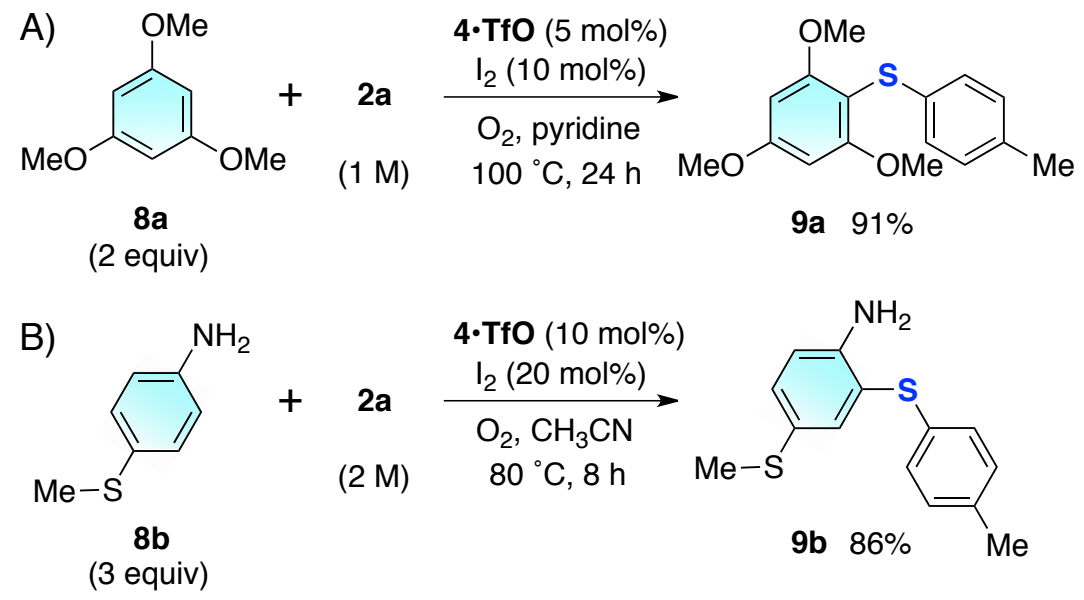


\section{2. ${ }^{1} \mathrm{H}$ and ${ }^{13} \mathbf{C}\left\{{ }^{1} \mathrm{H}\right\}$ NMR Spectra of Novel Compounds}

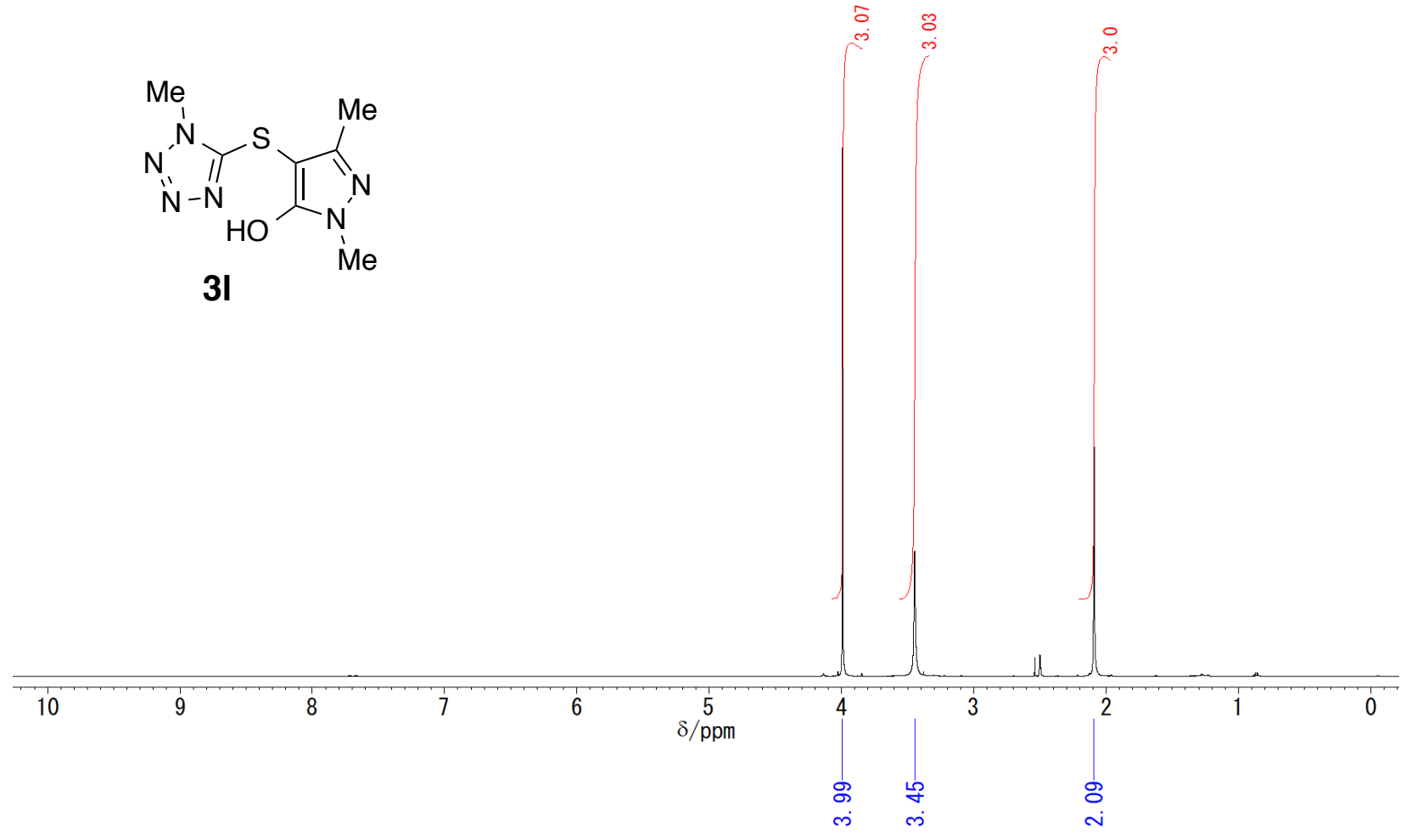

Spectrum S1. ${ }^{1} \mathrm{H}$ NMR (DMSO- $d_{6}, 500 \mathrm{MHz}$ ) spectrum of compound 31 .

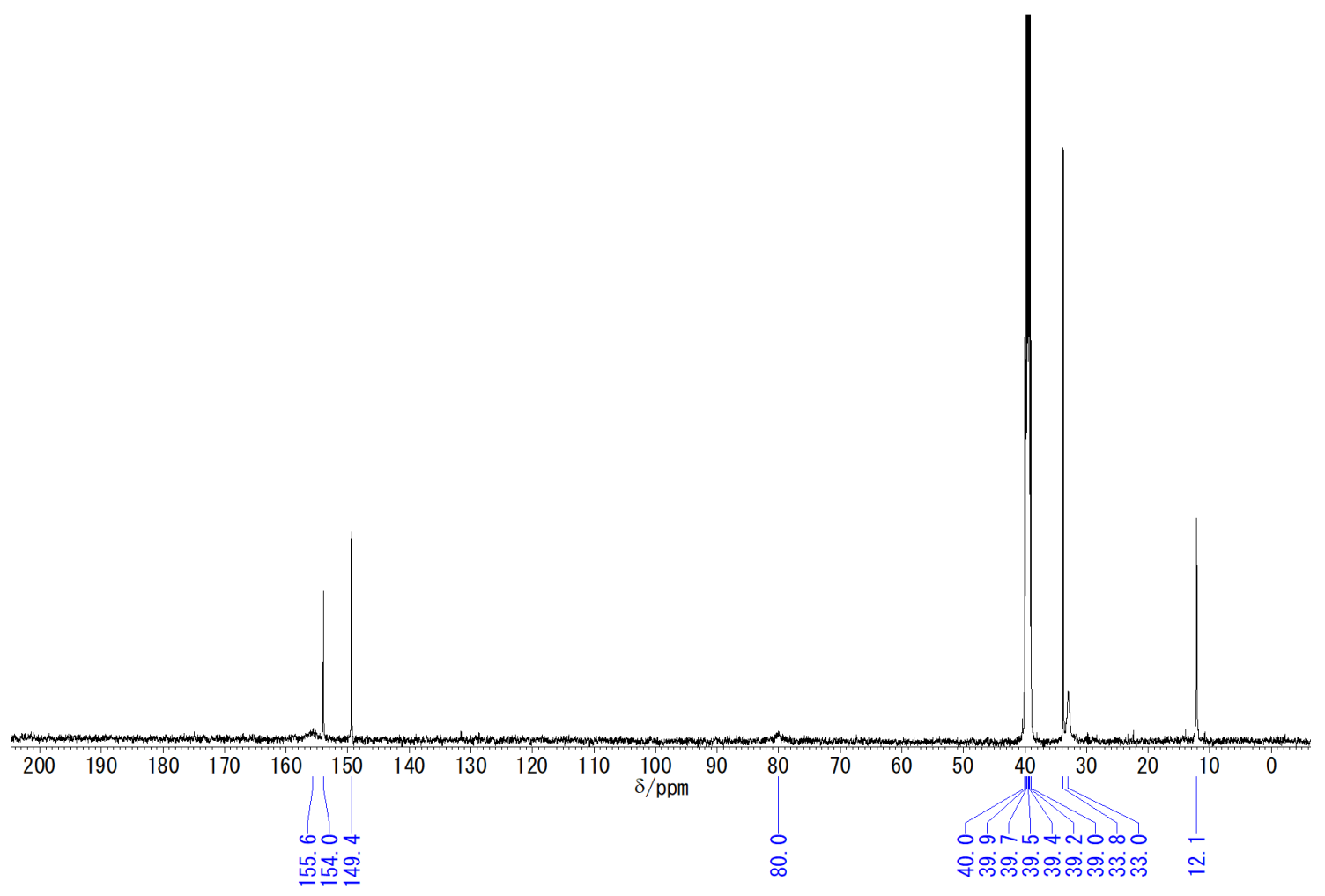

Spectrum S2. ${ }^{13} \mathrm{C}\left\{{ }^{1} \mathrm{H}\right\}$ NMR (DMSO- $d_{6}, 126 \mathrm{MHz}$ ) spectrum of compound 31 . 


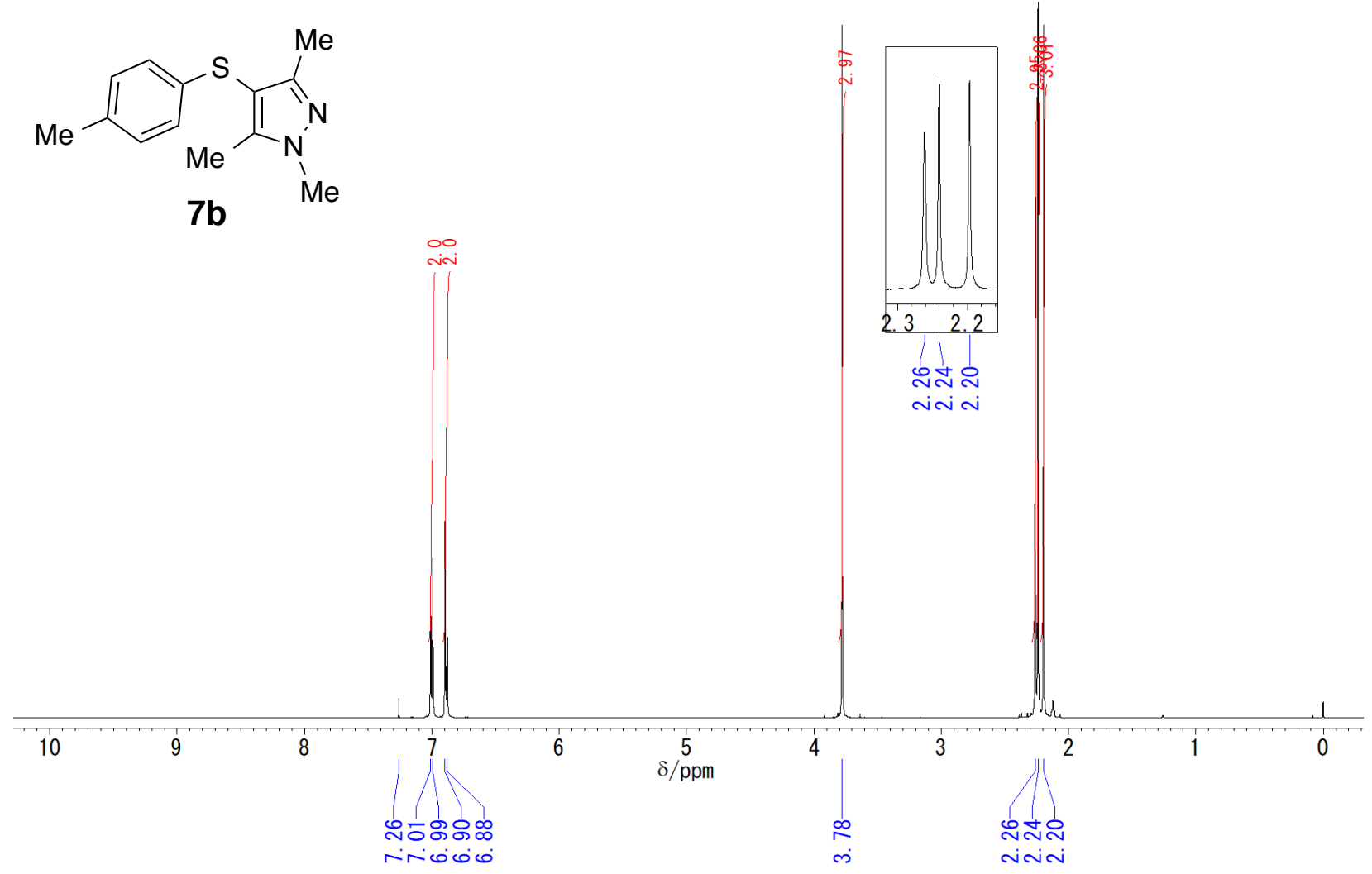

Spectrum S3. ${ }^{1} \mathrm{H}$ NMR $\left(\mathrm{CDCl}_{3}, 500 \mathrm{MHz}\right)$ spectrum of compound $7 \mathbf{b}$.

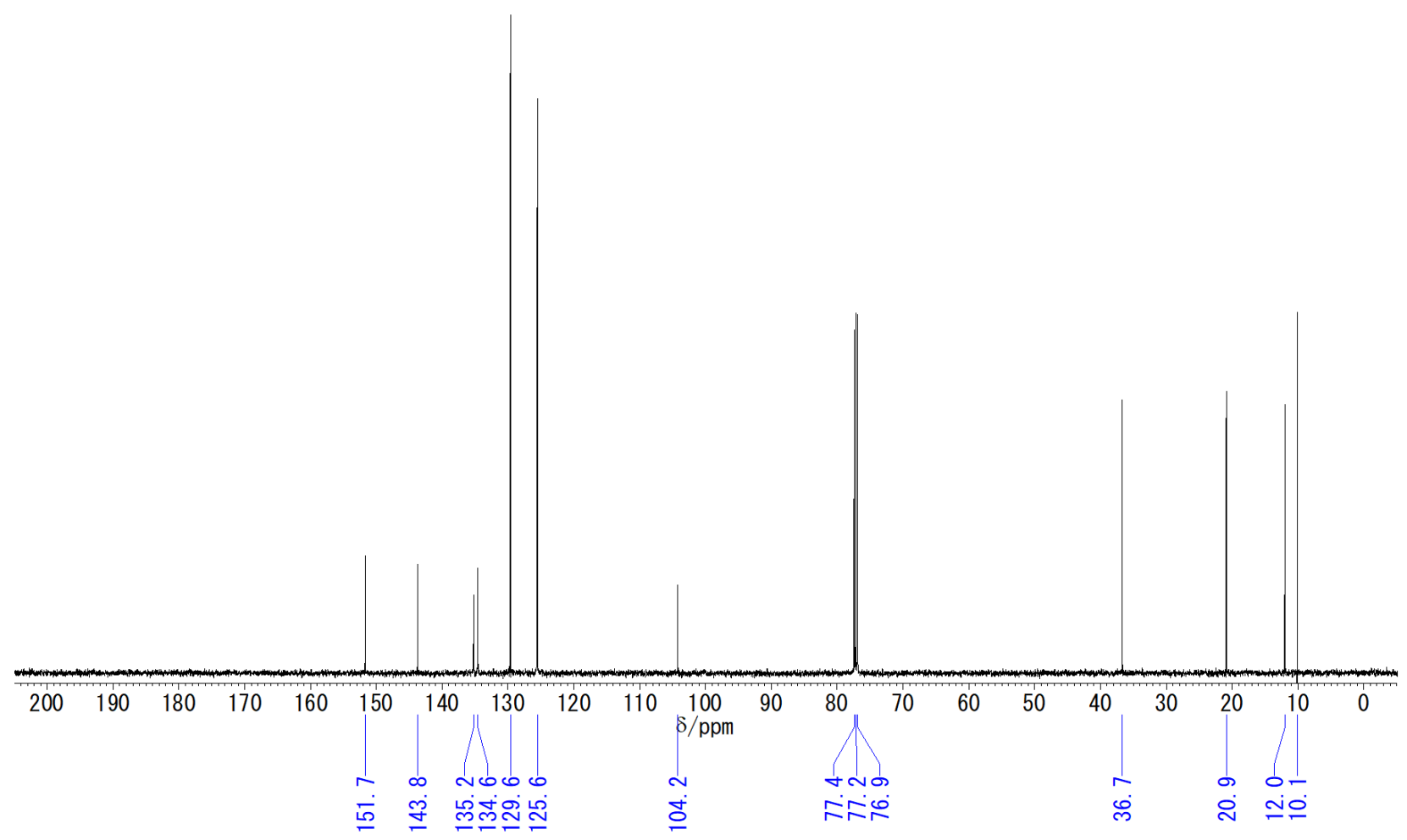

Spectrum S4. ${ }^{13} \mathrm{C}\left\{{ }^{1} \mathrm{H}\right\}$ NMR $\left(\mathrm{CDCl}_{3}, 126 \mathrm{MHz}\right)$ spectrum of compound $\mathbf{7 b}$. 


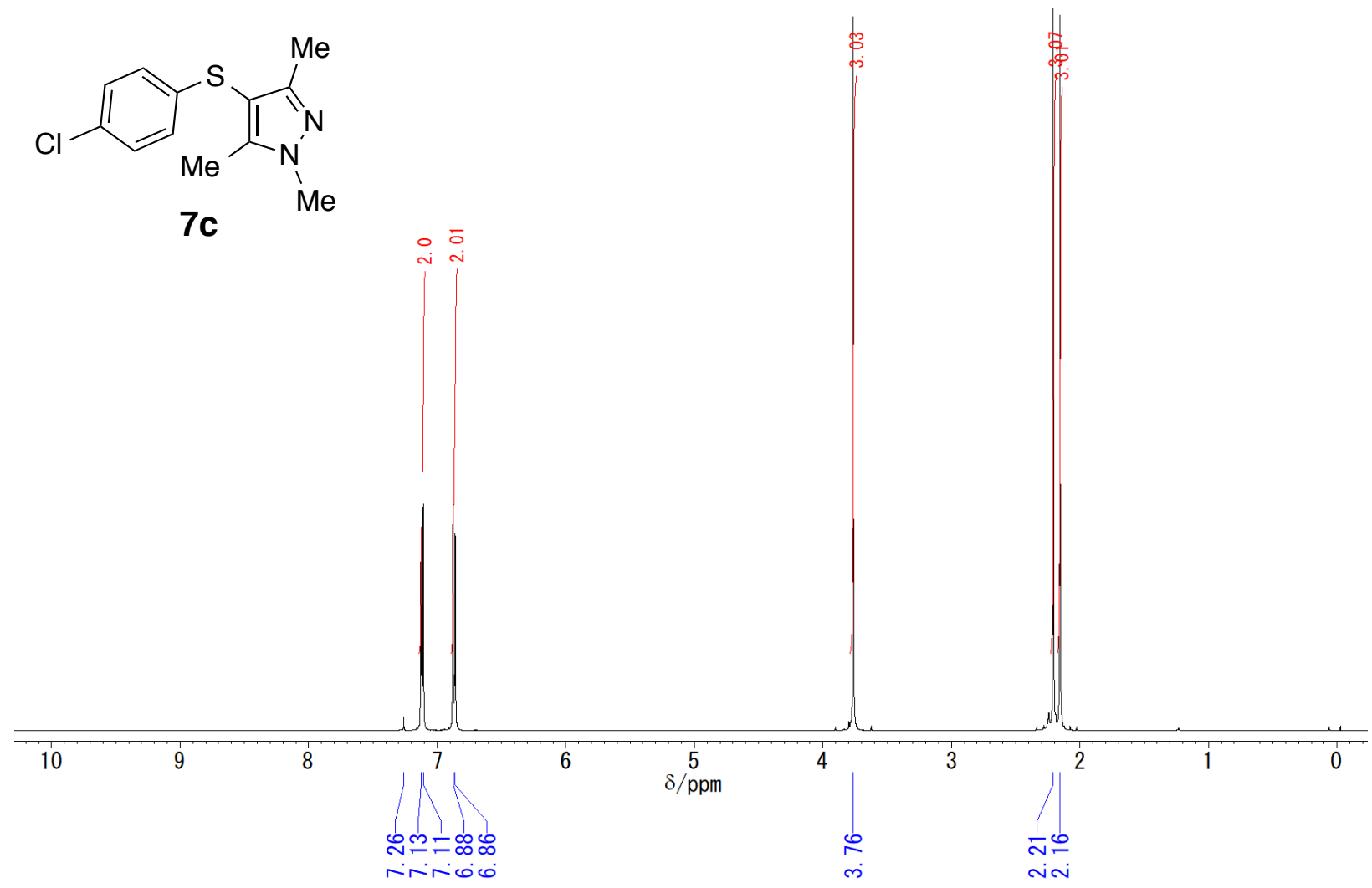

Spectrum S5. ${ }^{1} \mathrm{H} \mathrm{NMR}\left(\mathrm{CDCl}_{3}, 500 \mathrm{MHz}\right)$ spectrum of compound 7c.

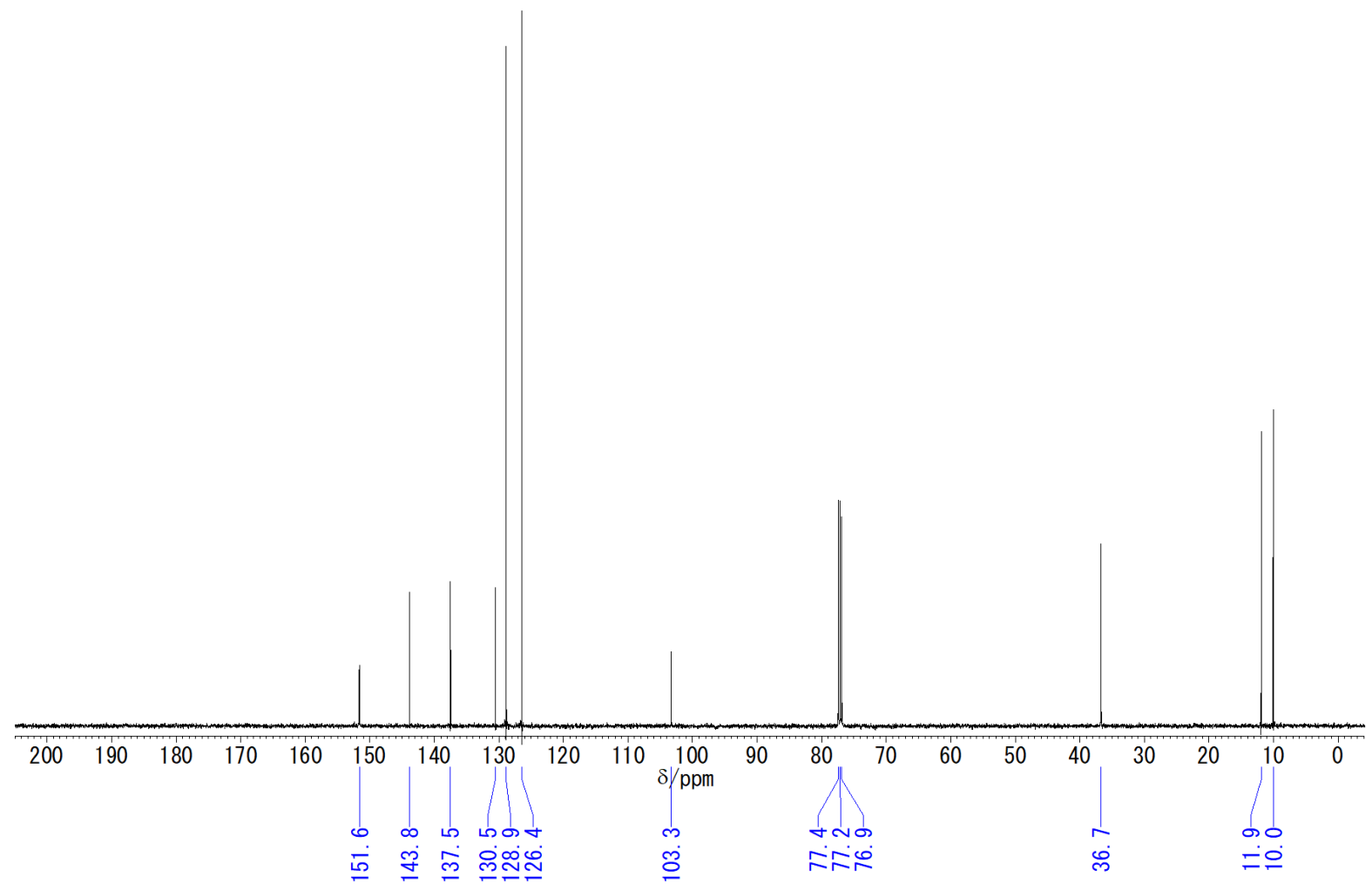

Spectrum S6. ${ }^{13} \mathrm{C}\left\{{ }^{1} \mathrm{H}\right\}$ NMR $\left(\mathrm{CDCl}_{3}, 126 \mathrm{MHz}\right)$ spectrum of compound 7c. 


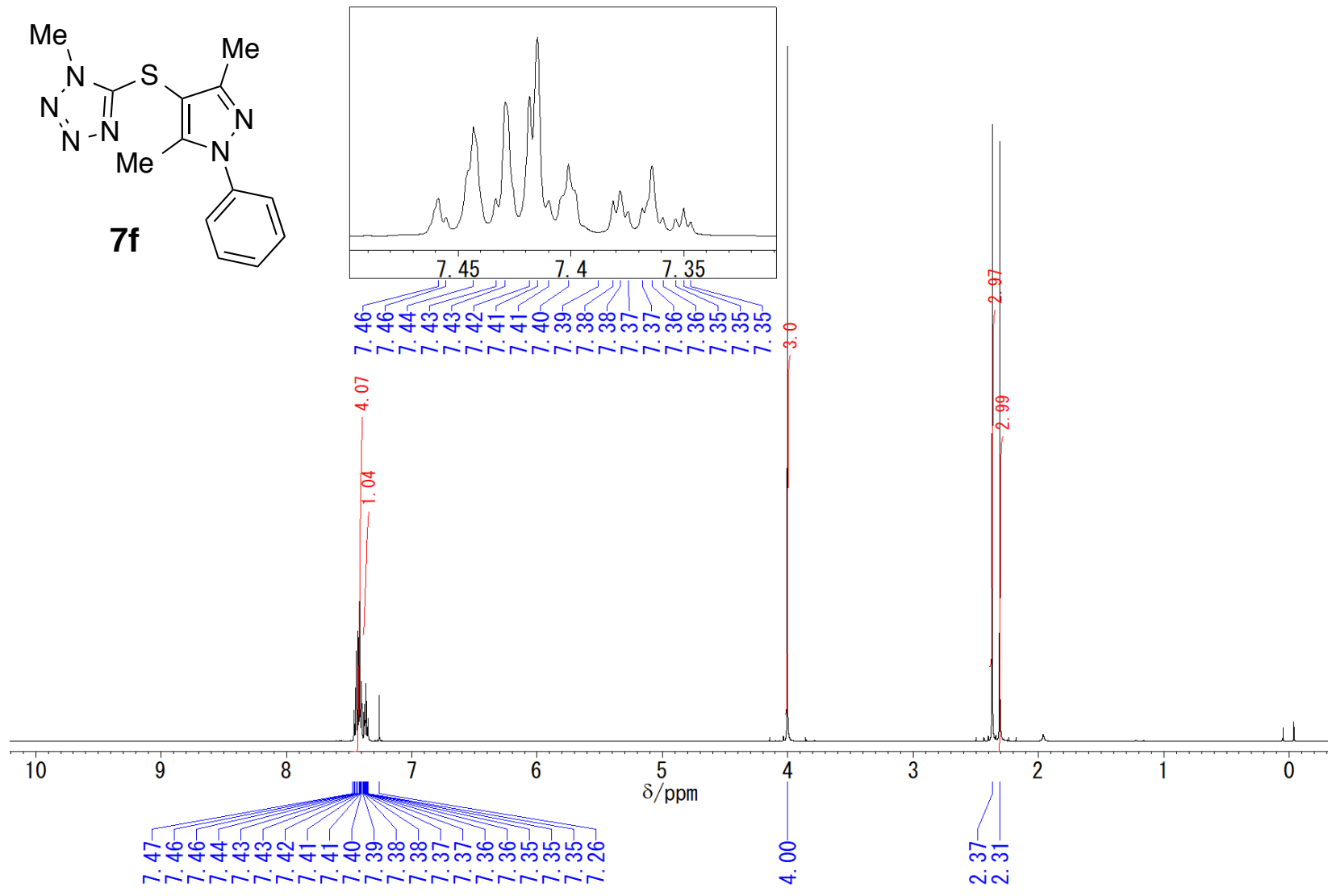

Spectrum S7. ${ }^{1} \mathrm{H} \mathrm{NMR}\left(\mathrm{CDCl}_{3}, 500 \mathrm{MHz}\right)$ spectrum of compound $7 \mathbf{f}$.

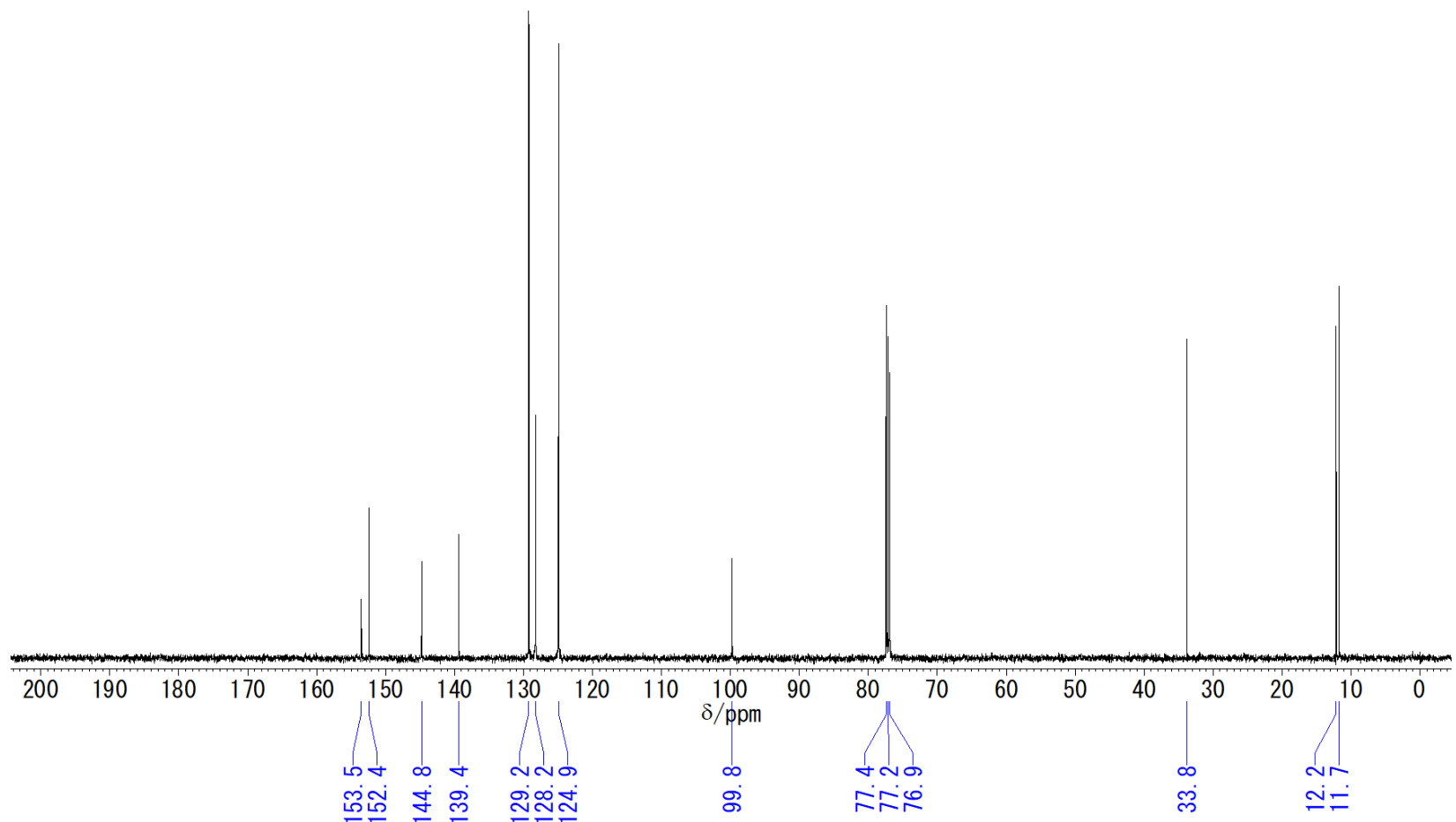

Spectrum S8. ${ }^{13} \mathrm{C}\left\{{ }^{1} \mathrm{H}\right\}$ NMR $\left(\mathrm{CDCl}_{3}, 126 \mathrm{MHz}\right)$ spectrum of compound $7 \mathbf{f}$. 


\section{3. ${ }^{1} \mathrm{H}$ and ${ }^{13} \mathrm{C}$ NMR Spectra of Known Compounds}

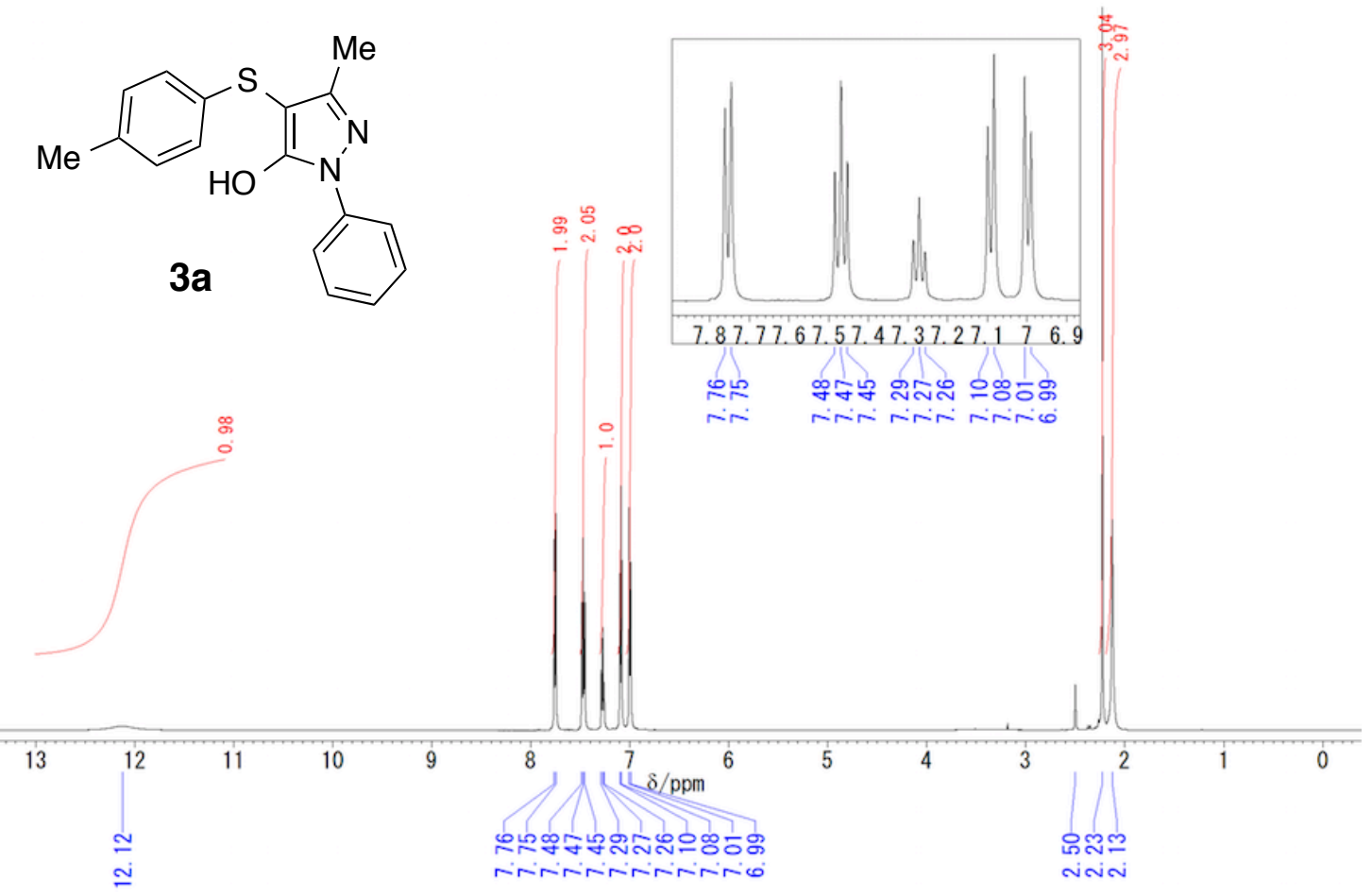

Spectrum S9. ${ }^{1} \mathrm{H}$ NMR (DMSO- $d_{6}, 500 \mathrm{MHz}$ ) spectrum of compound $3 \mathbf{a}$.

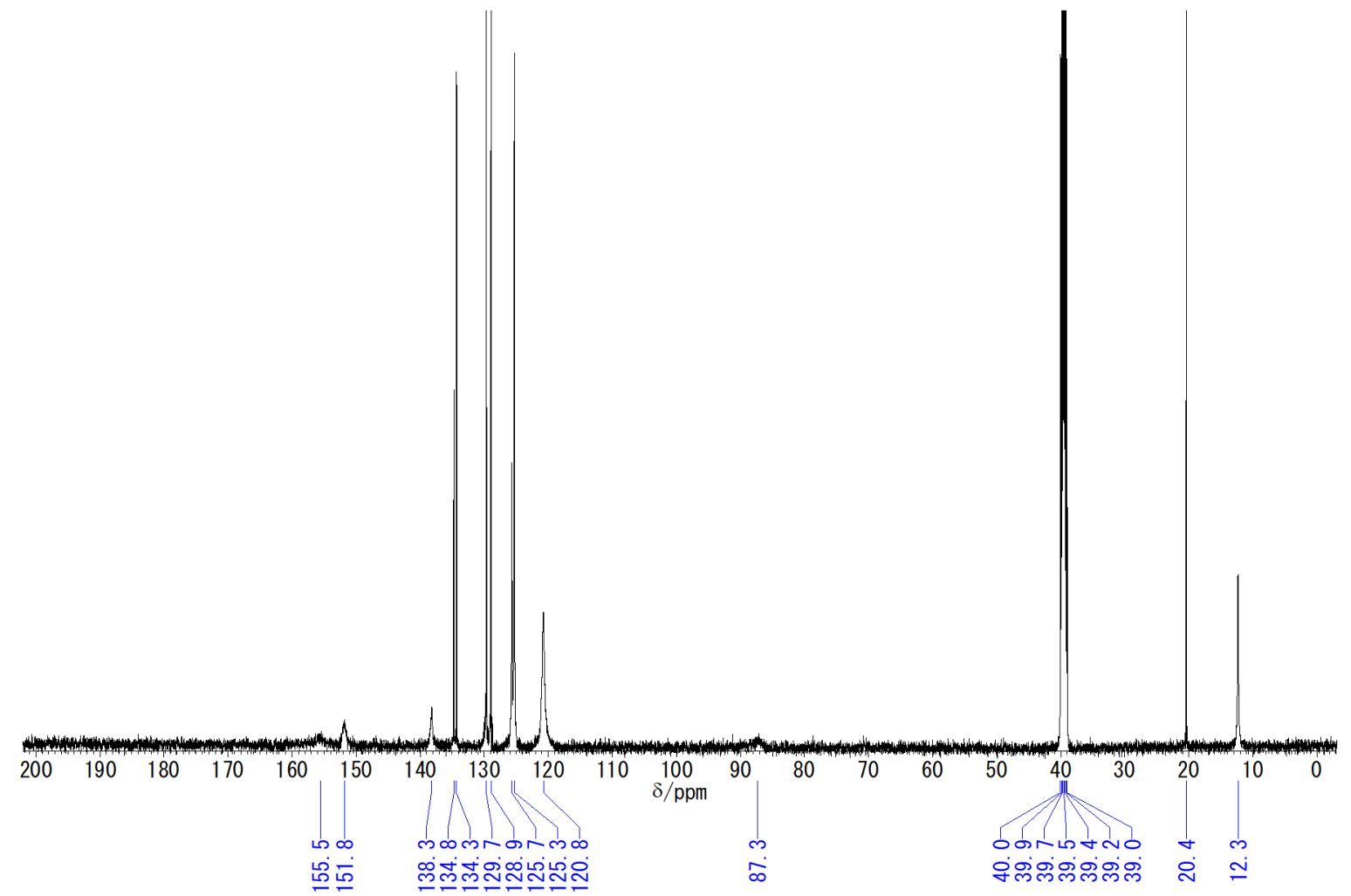

Spectrum S10. ${ }^{13} \mathrm{C}\left\{{ }^{1} \mathrm{H}\right\}$ NMR (DMSO- $d_{6}, 126 \mathrm{MHz}$ ) spectrum of compound 3a. 


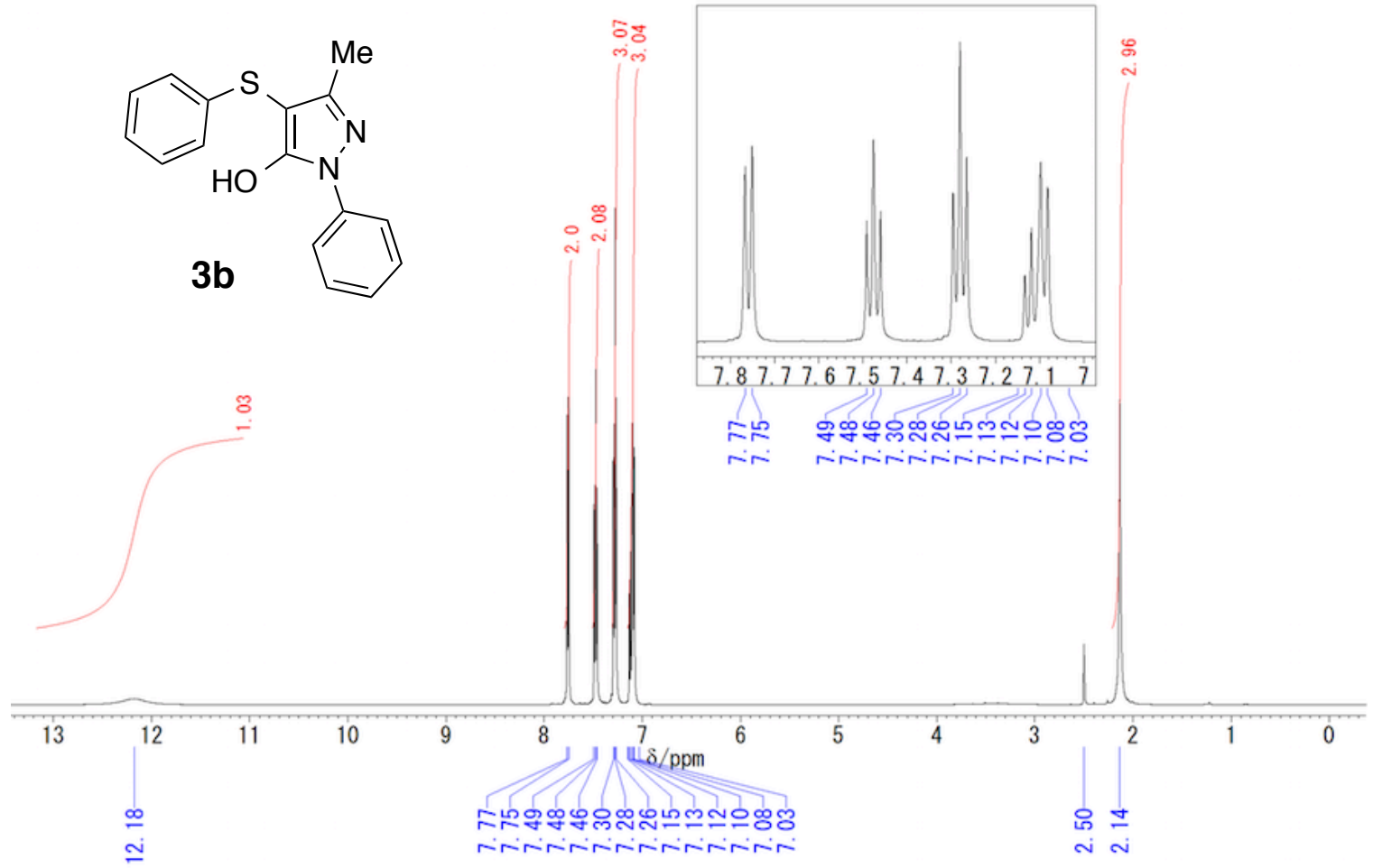

Spectrum S11. ${ }^{1} \mathrm{H}$ NMR (DMSO- $\left.d_{6}, 500 \mathrm{MHz}\right)$ spectrum of compound $\mathbf{3 b}$.

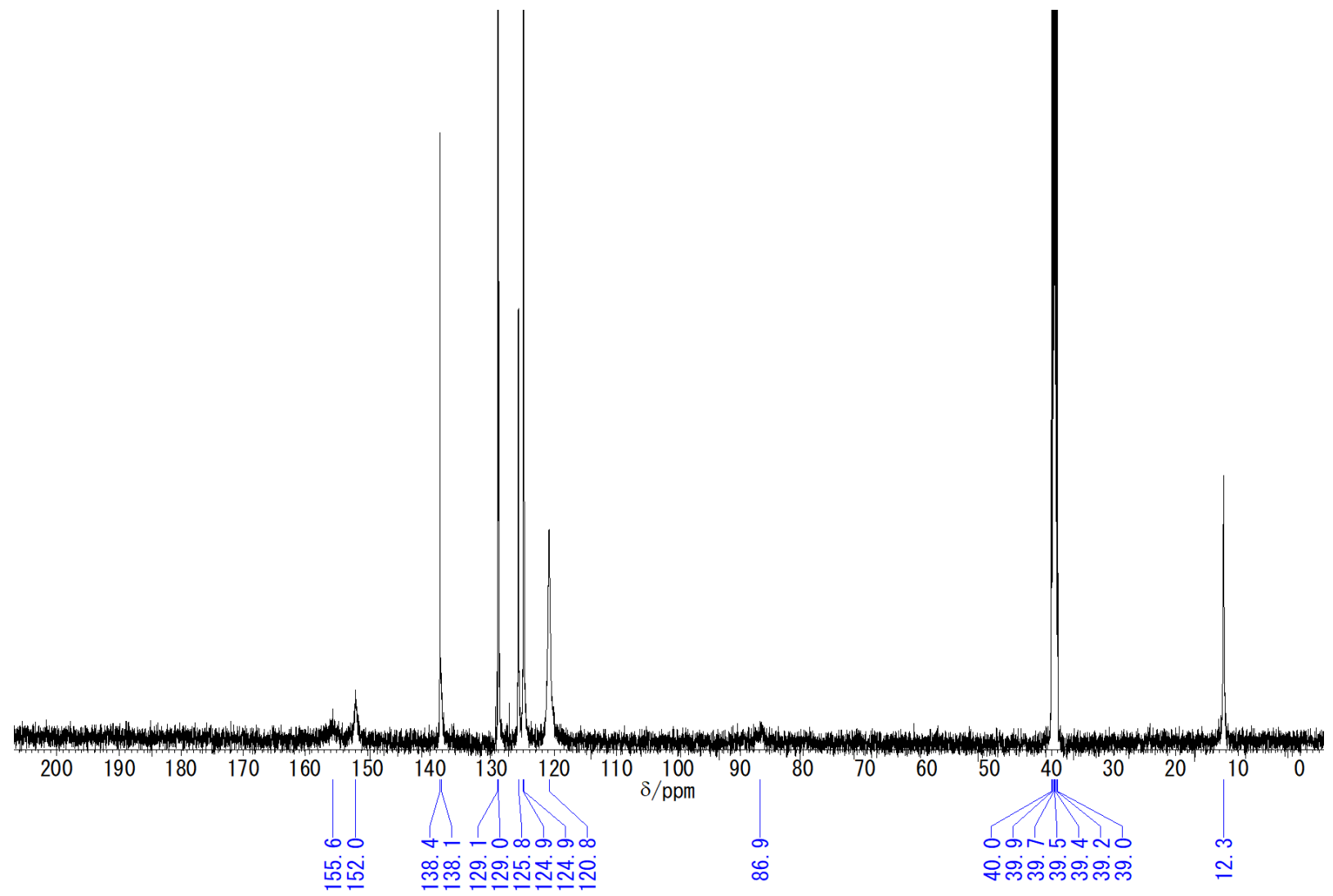

Spectrum S12. ${ }^{13} \mathrm{C}\left\{{ }^{1} \mathrm{H}\right\}$ NMR (DMSO- $d_{6}, 126 \mathrm{MHz}$ ) spectrum of compound $\mathbf{3 b}$. 


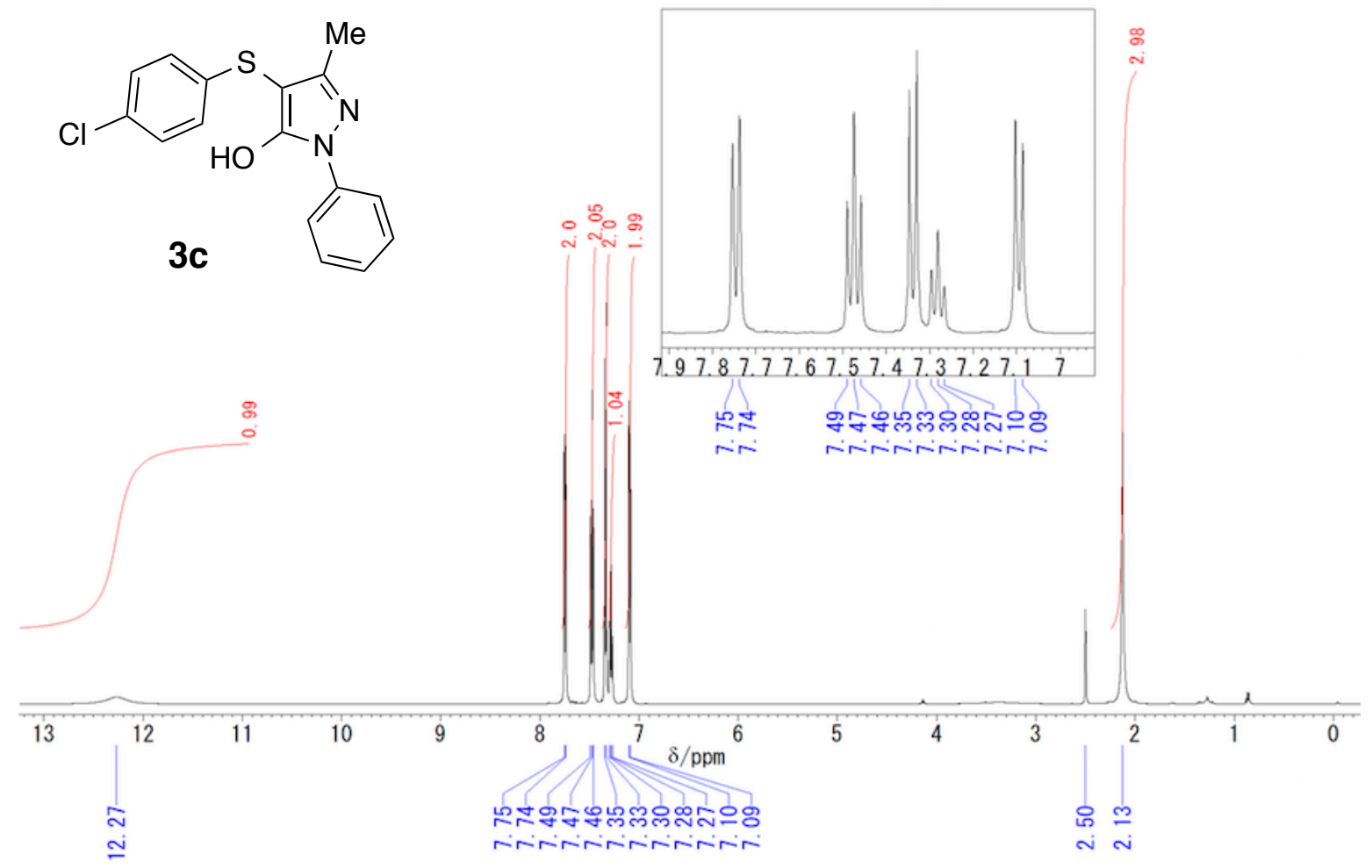

Spectrum S13. ${ }^{1} \mathrm{H}$ NMR (DMSO- $d_{6}, 500 \mathrm{MHz}$ ) spectrum of compound 3c.

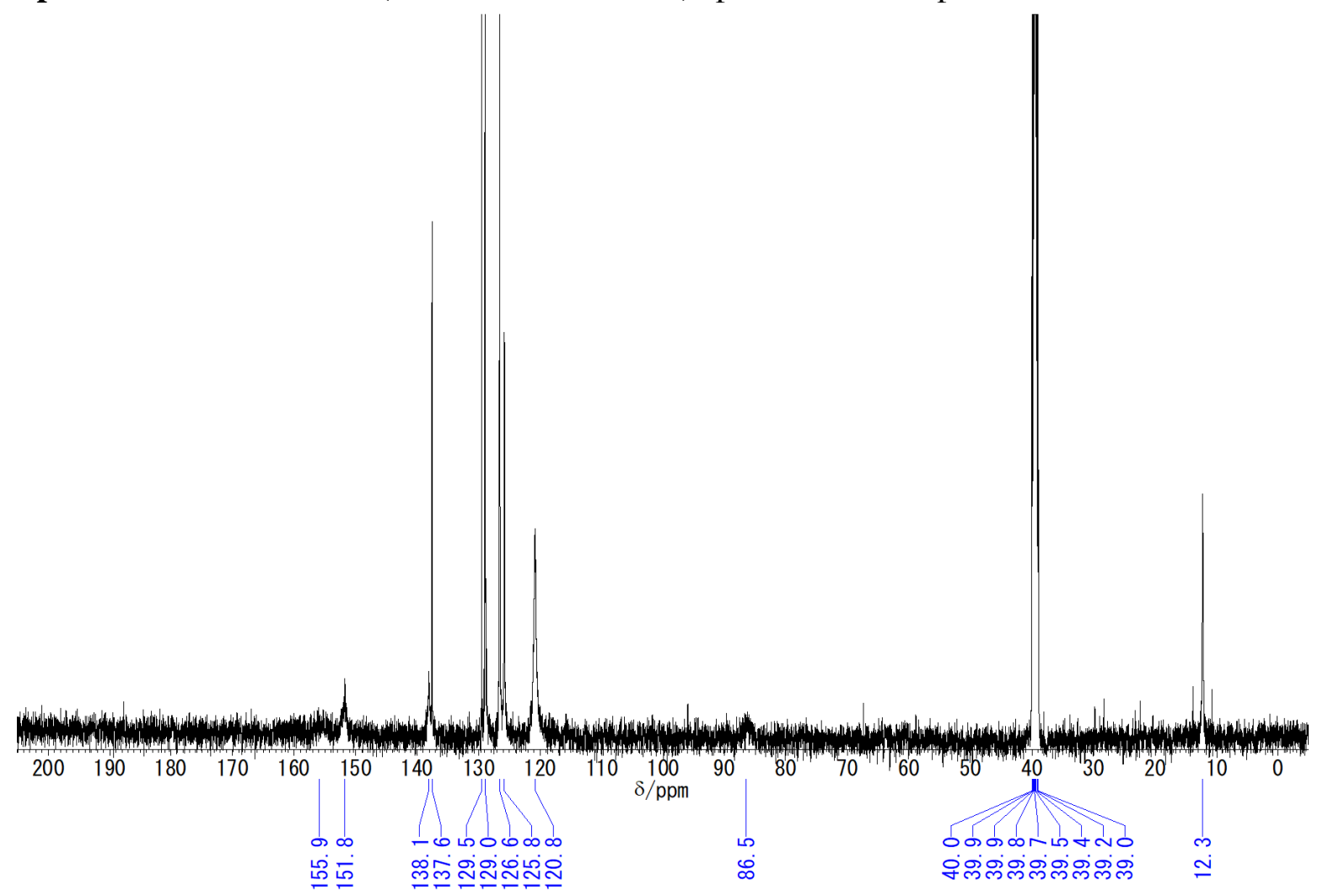

Spectrum S14. ${ }^{13} \mathrm{C}\left\{{ }^{1} \mathrm{H}\right\}$ NMR (DMSO- $d_{6}, 126 \mathrm{MHz}$ ) spectrum of compound 3c. 


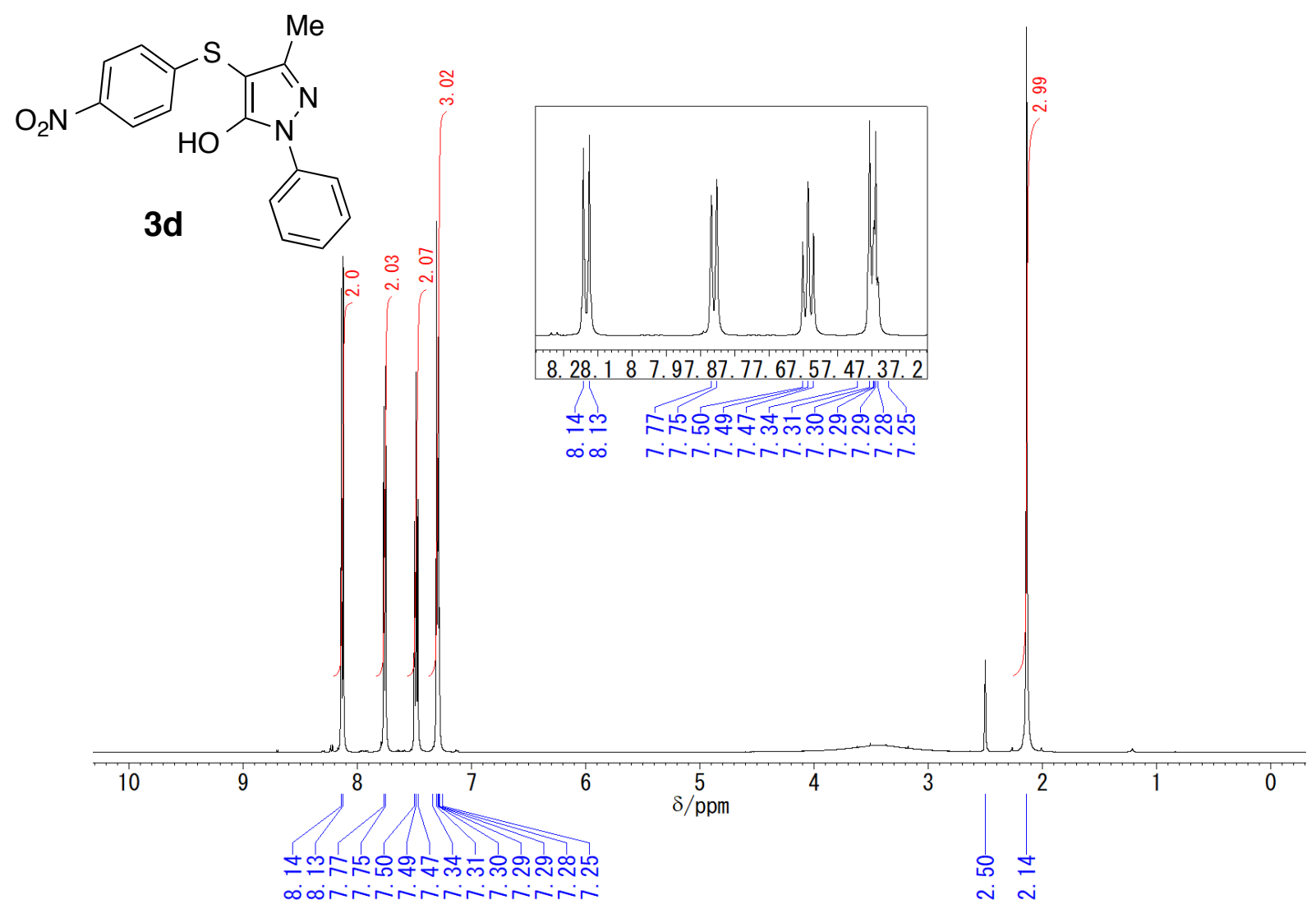

Spectrum S15. ${ }^{1} \mathrm{H}$ NMR (DMSO- $d_{6}, 500 \mathrm{MHz}$ ) spectrum of compound 3d.

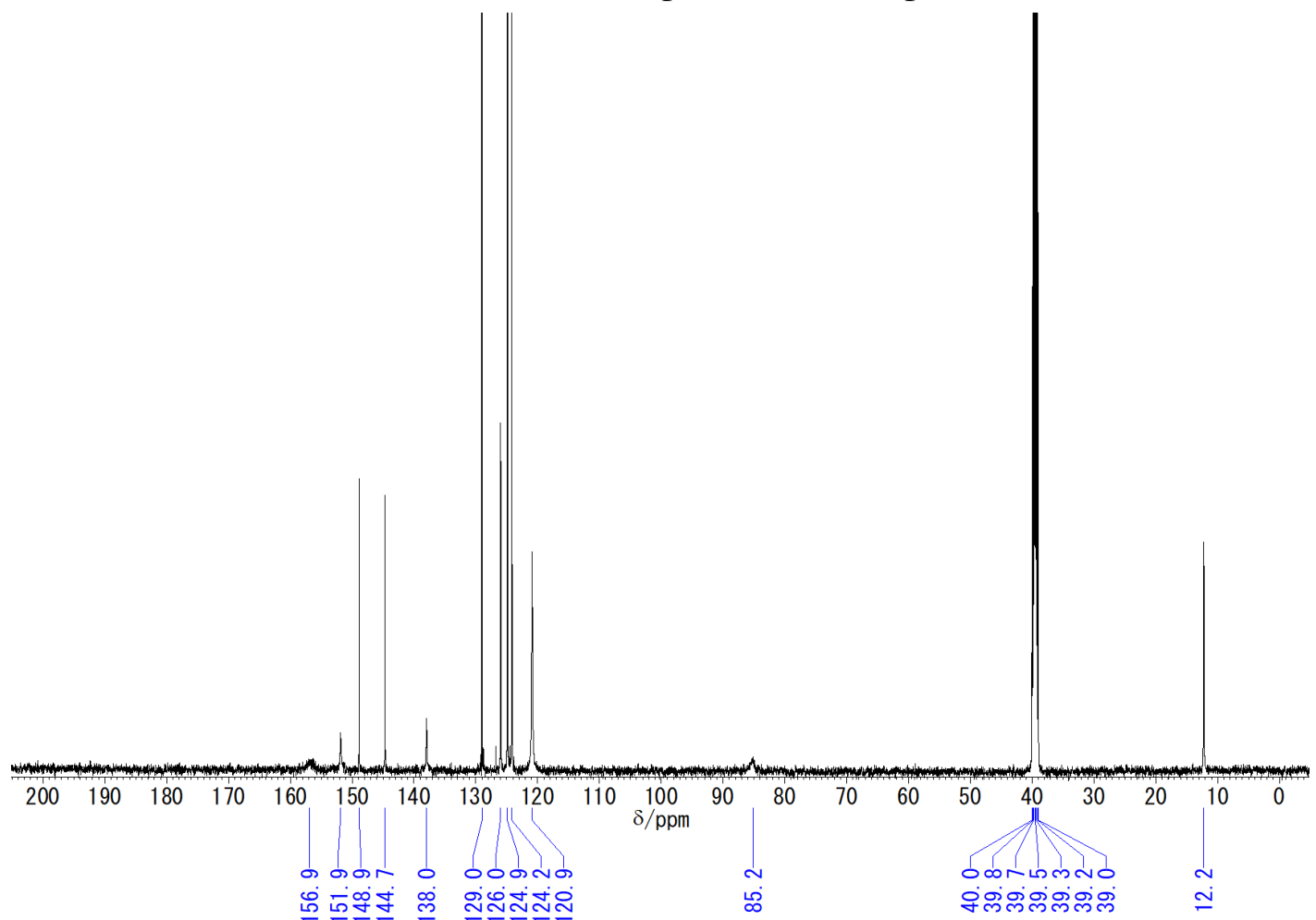

Spectrum S16. ${ }^{13} \mathrm{C}\left\{{ }^{1} \mathrm{H}\right\}$ NMR (DMSO- $d_{6}, 126 \mathrm{MHz}$ ) spectrum of compound 3d. 


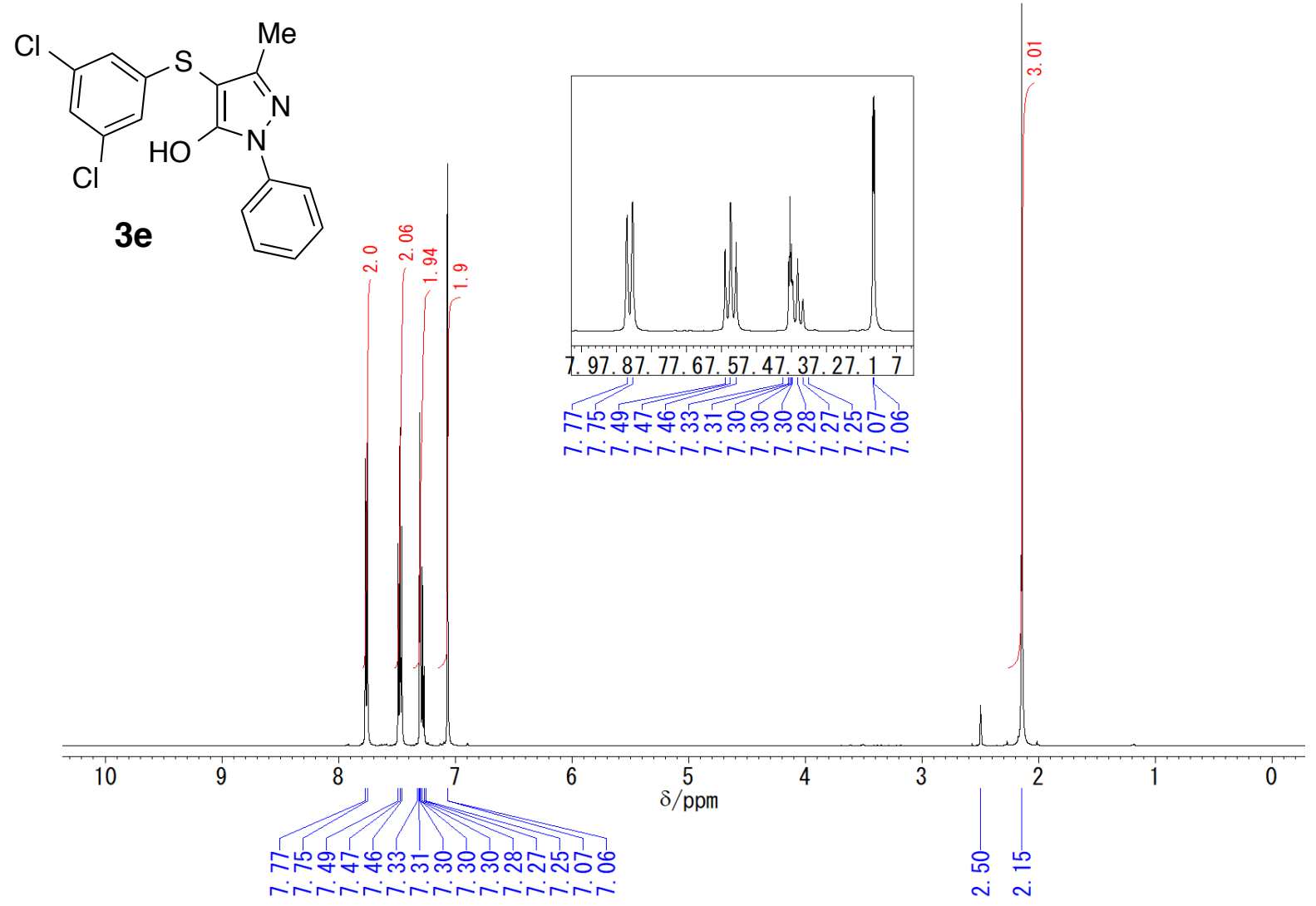

Spectrum S17. ${ }^{1} \mathrm{H}$ NMR (DMSO- $d_{6}, 500 \mathrm{MHz}$ ) spectrum of compound 3e.

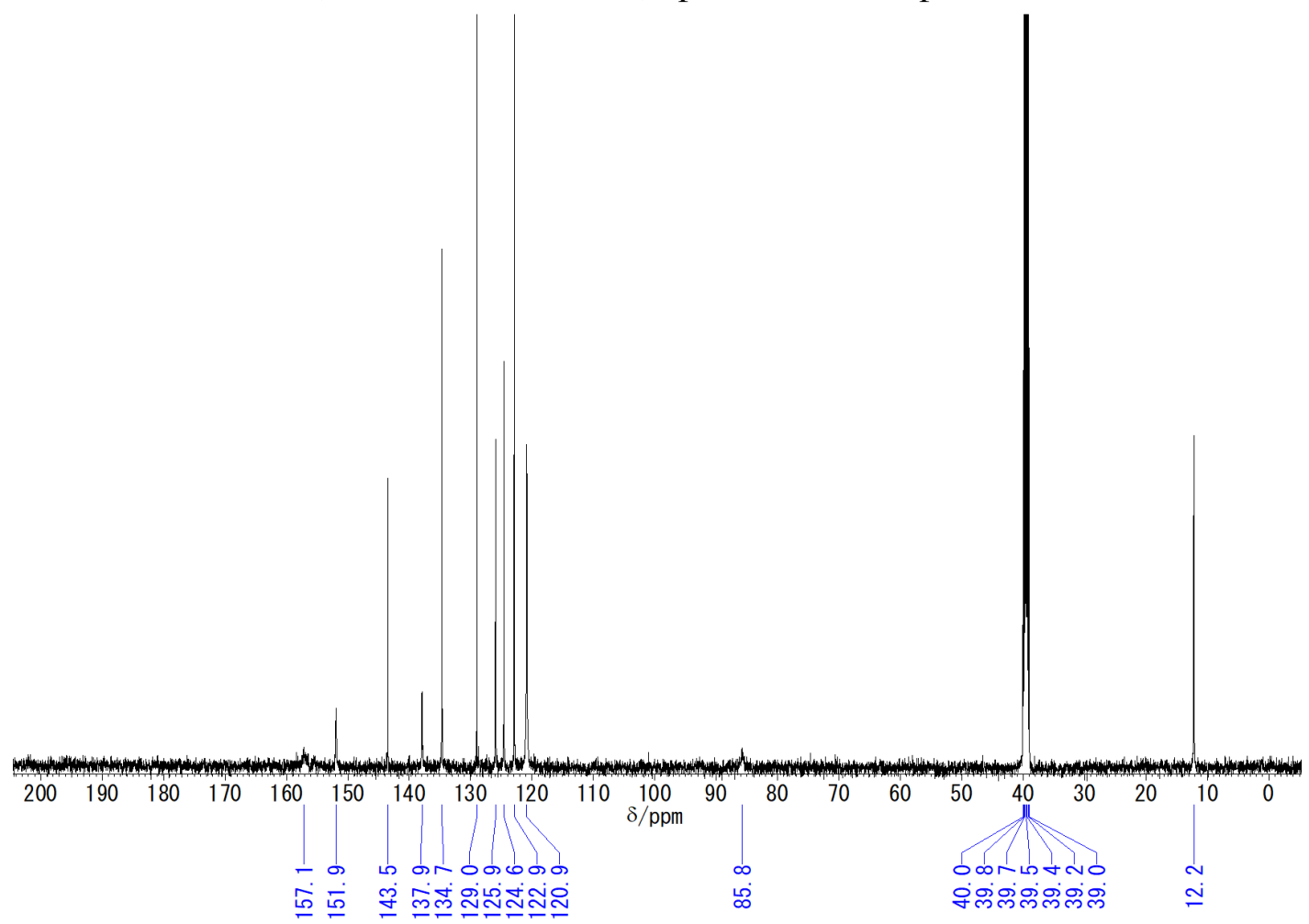

Spectrum S18. ${ }^{13} \mathrm{C}\left\{{ }^{1} \mathrm{H}\right\}$ NMR (DMSO- $d_{6}, 126 \mathrm{MHz}$ ) spectrum of compound 3e. 


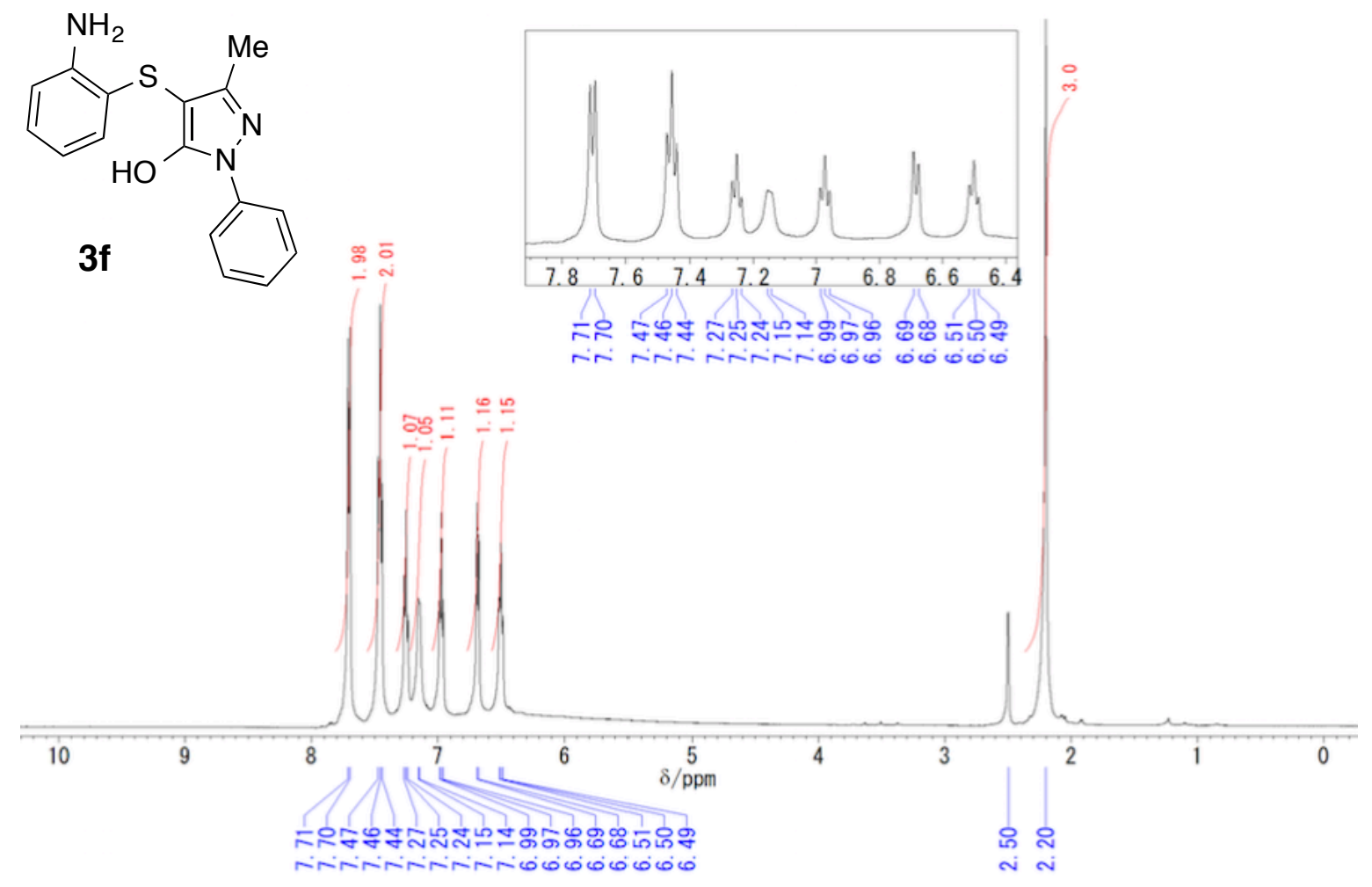

Spectrum S19. ${ }^{1} \mathrm{H}$ NMR (DMSO- $d_{6}, 500 \mathrm{MHz}$ ) spectrum of compound $3 \mathrm{f}$.

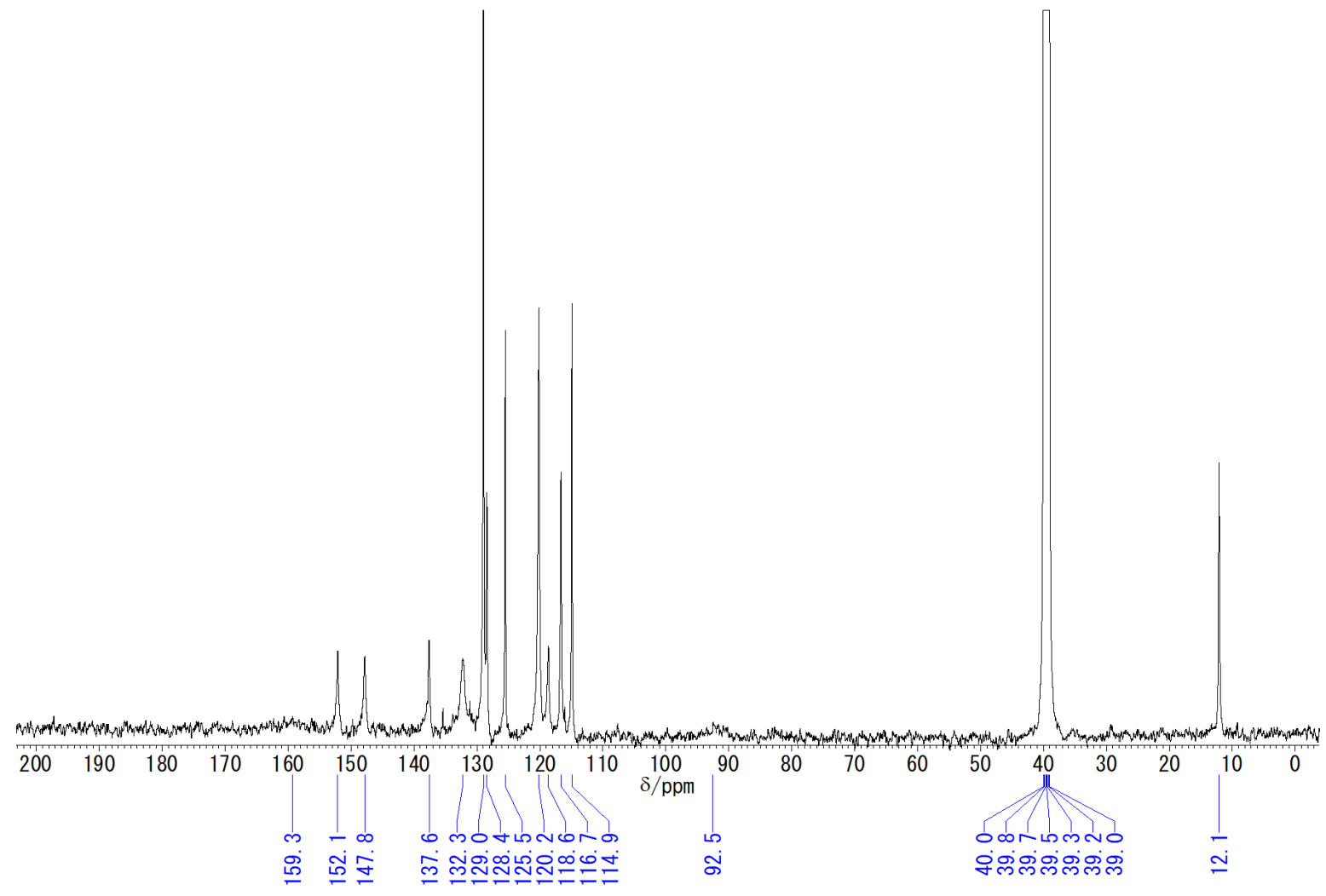

Spectrum S20. ${ }^{13} \mathrm{C}\left\{{ }^{1} \mathrm{H}\right\}$ NMR (DMSO- $d_{6}, 126 \mathrm{MHz}$ ) spectrum of compound 3f. 


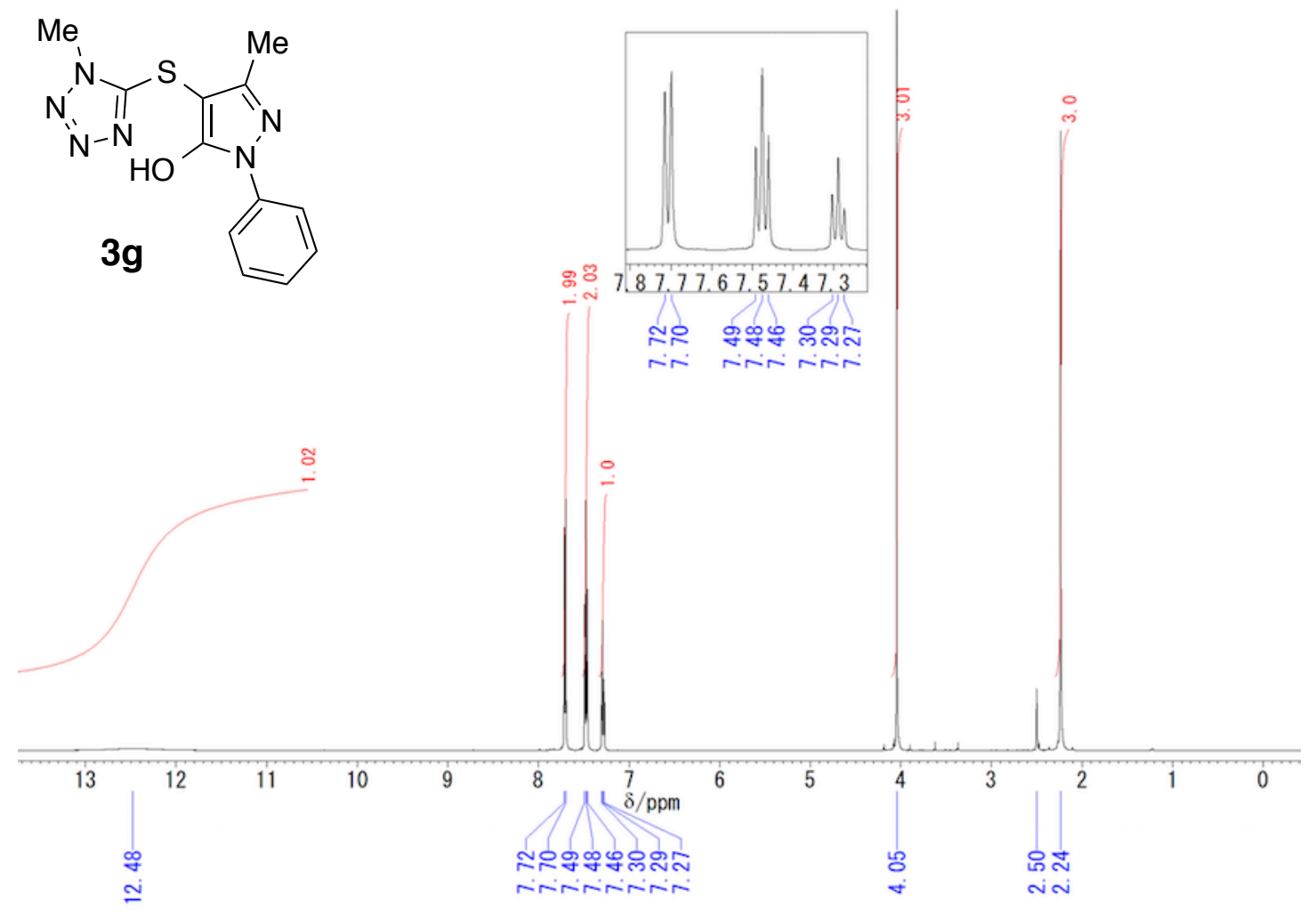

Spectrum S21. ${ }^{1} \mathrm{H}$ NMR (DMSO- $d_{6}, 500 \mathrm{MHz}$ ) spectrum of compound 3g.

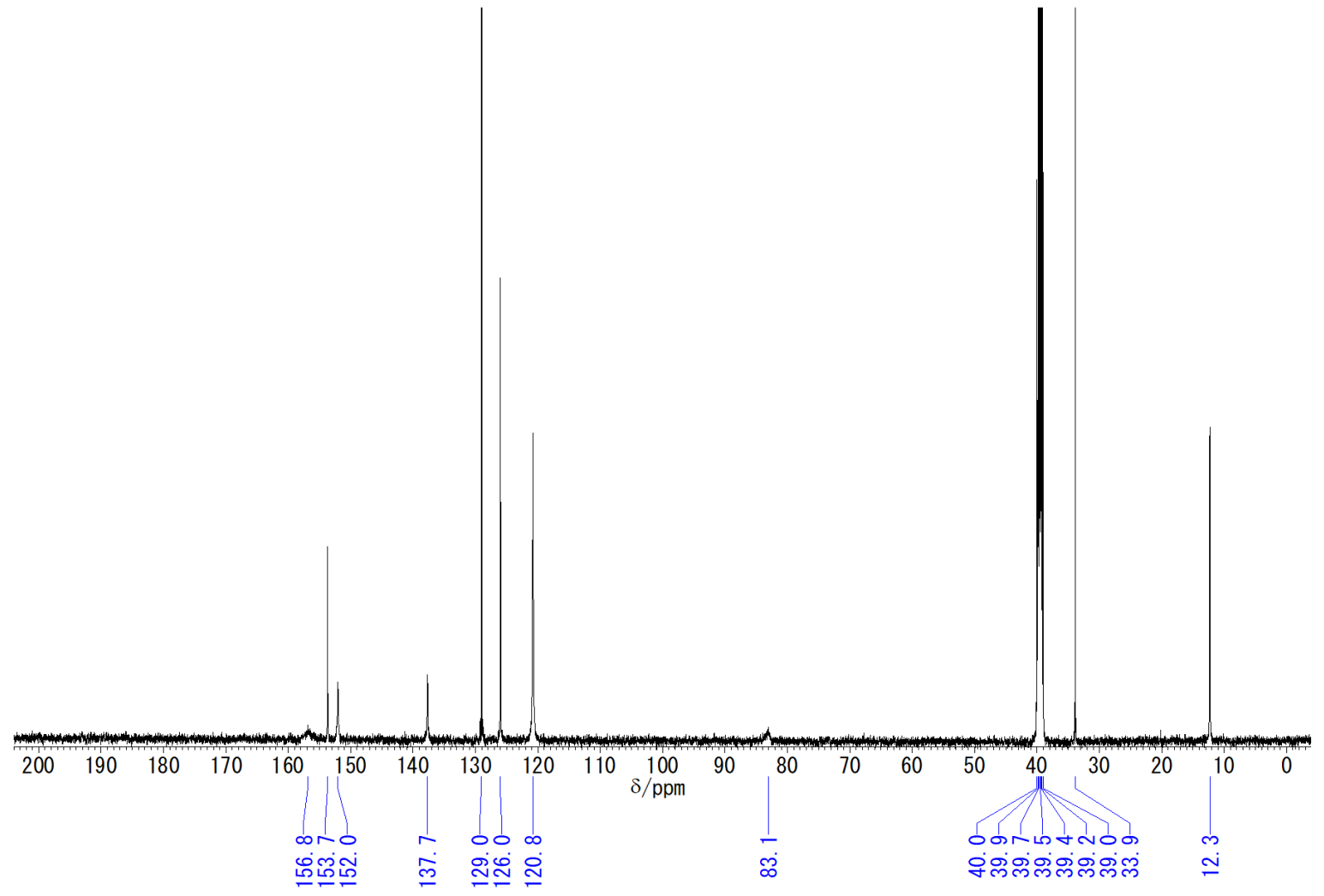

Spectrum S22. ${ }^{13} \mathrm{C}\left\{{ }^{1} \mathrm{H}\right\}$ NMR (DMSO- $d_{6}, 126 \mathrm{MHz}$ ) spectrum of compound 3g. 


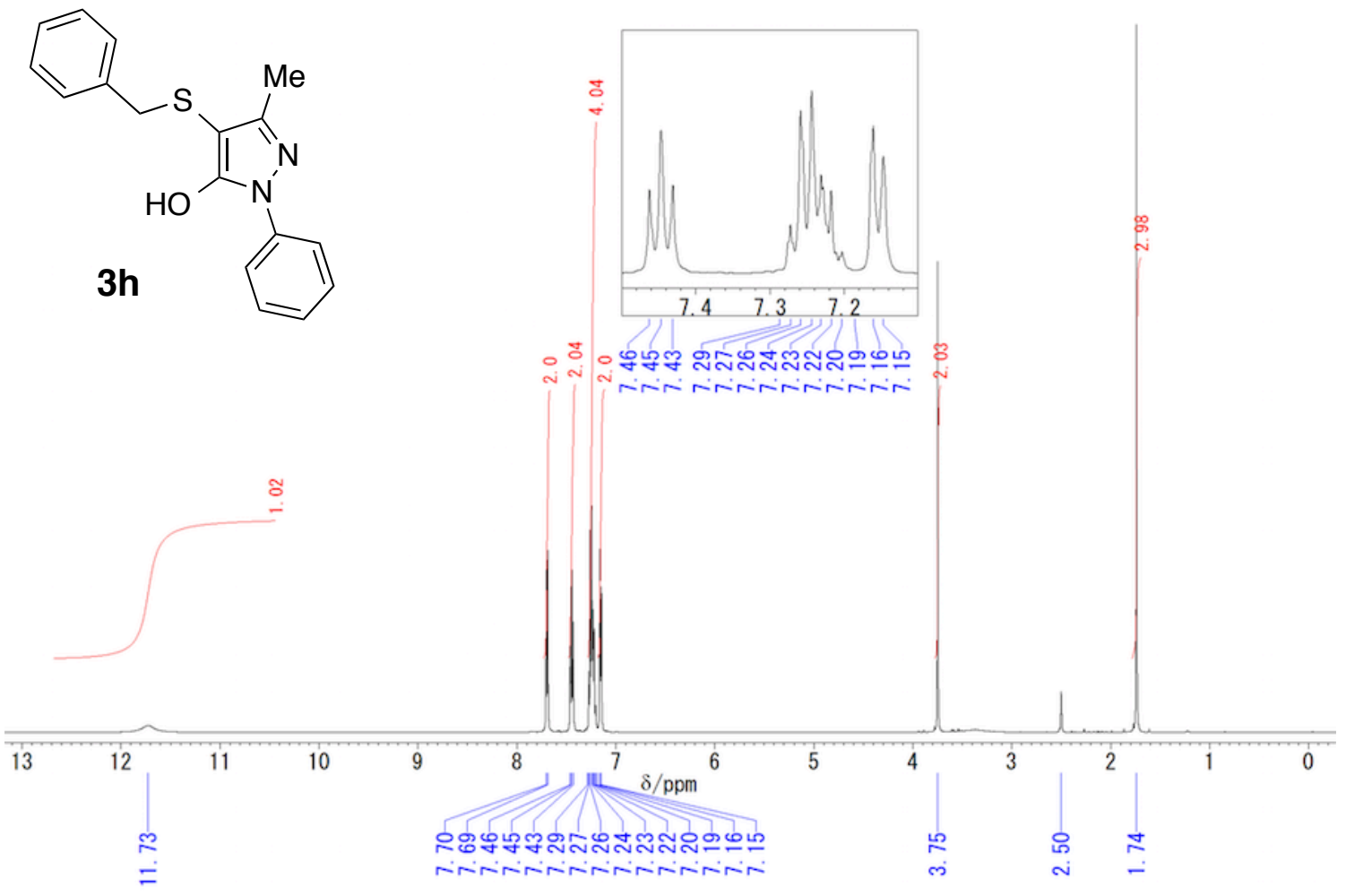

Spectrum S23. ${ }^{1} \mathrm{H}$ NMR (DMSO- $d_{6}, 500 \mathrm{MHz}$ ) spectrum of compound $\mathbf{3 h}$.

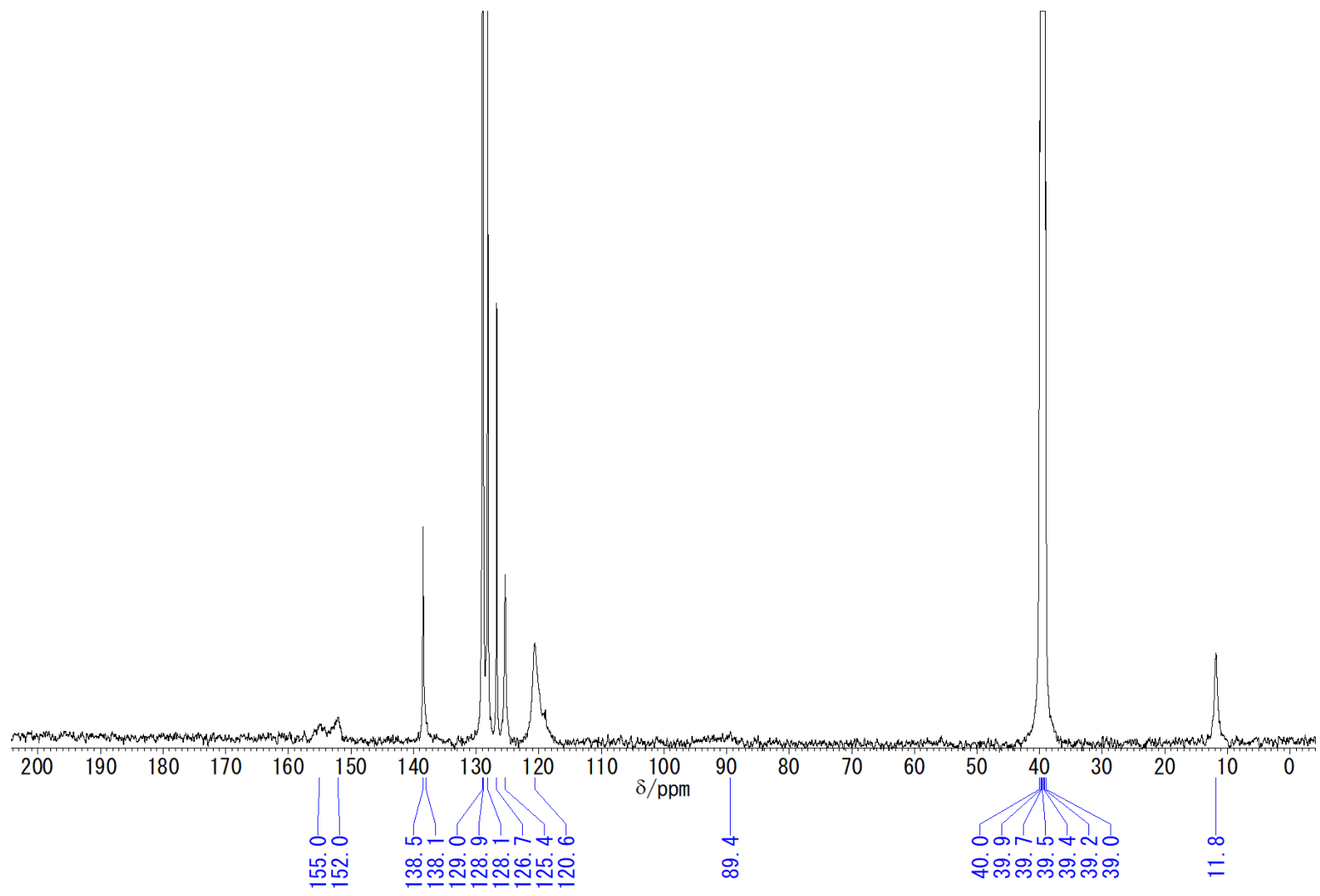

Spectrum S24. ${ }^{13} \mathrm{C}\left\{{ }^{1} \mathrm{H}\right\}$ NMR (DMSO- $d_{6}, 126 \mathrm{MHz}$ ) spectrum of compound $\mathbf{3 h}$. 


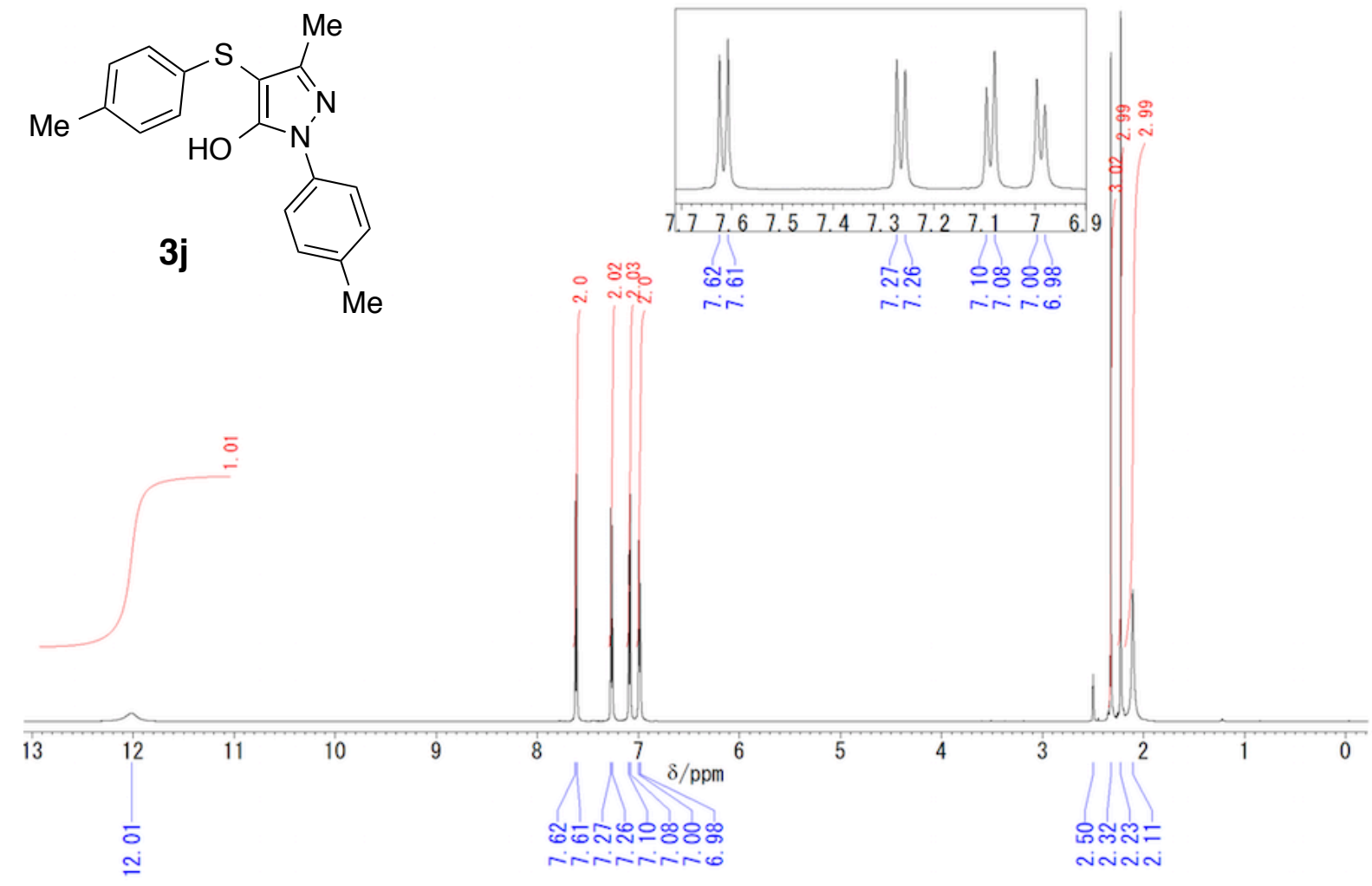

Spectrum S25. ${ }^{1} \mathrm{H}$ NMR (DMSO- $d_{6}, 500 \mathrm{MHz}$ ) spectrum of compound $\mathbf{3 j}$.

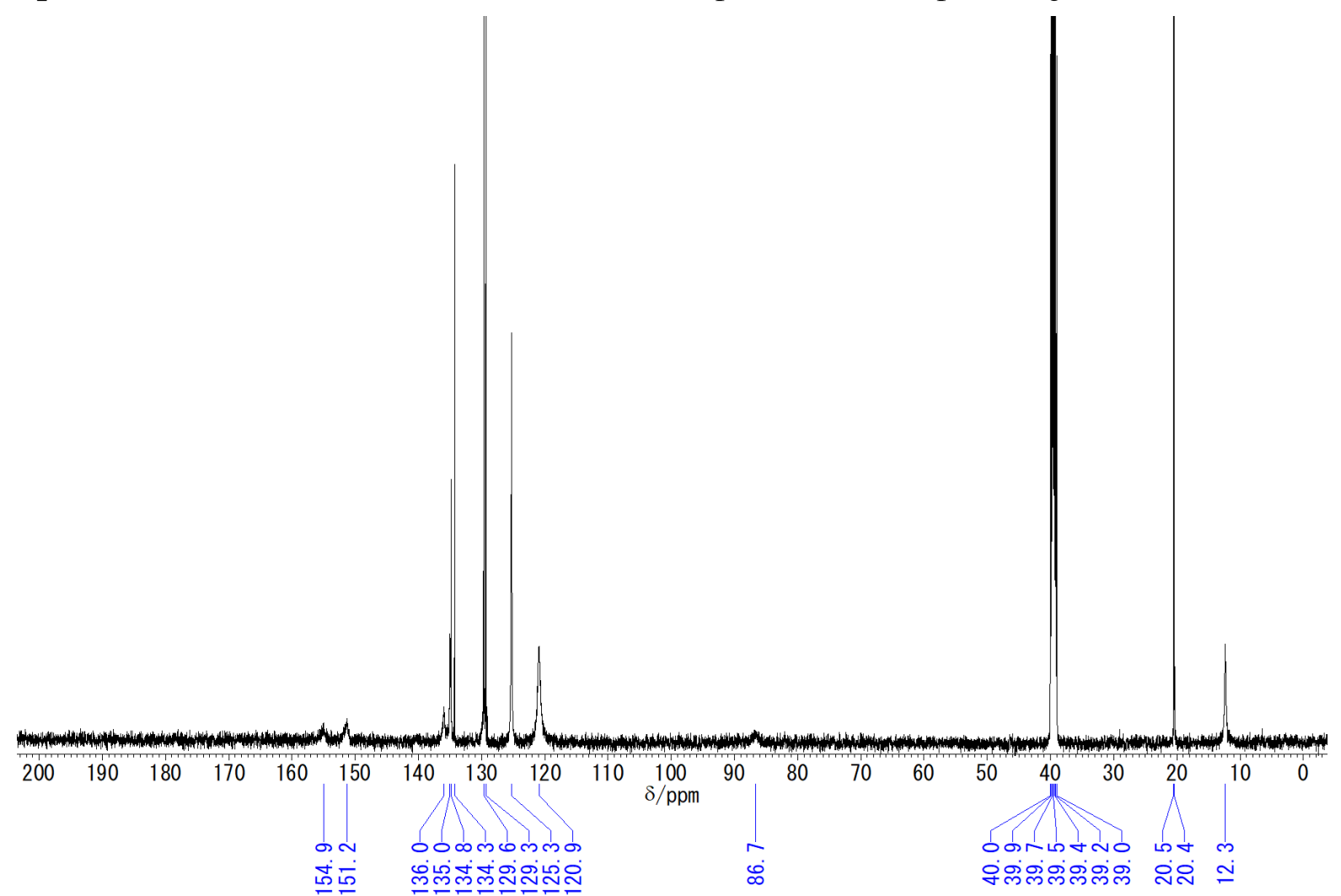

Spectrum S26. ${ }^{13} \mathrm{C}\left\{{ }^{1} \mathrm{H}\right\}$ NMR (DMSO- $d_{6}, 126 \mathrm{MHz}$ ) spectrum of compound 3j. 
<smiles>Cc1ccc(Sc2c(C)nn(-c3ccc(Cl)cc3)c2O)cc1</smiles>
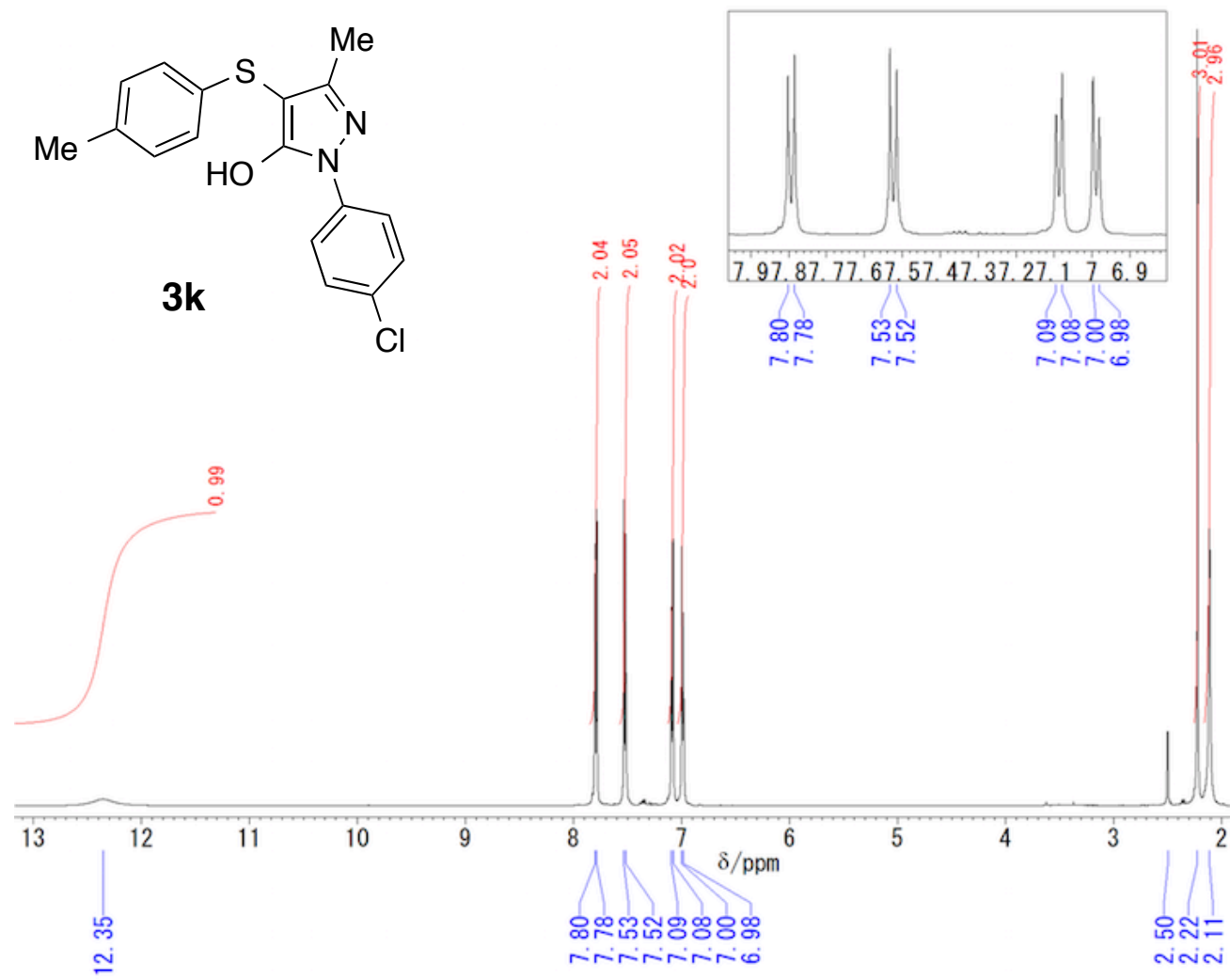

Spectrum S27. ${ }^{1} \mathrm{H}$ NMR (DMSO- $d_{6}, 500 \mathrm{MHz}$ ) spectrum of compound 3k.

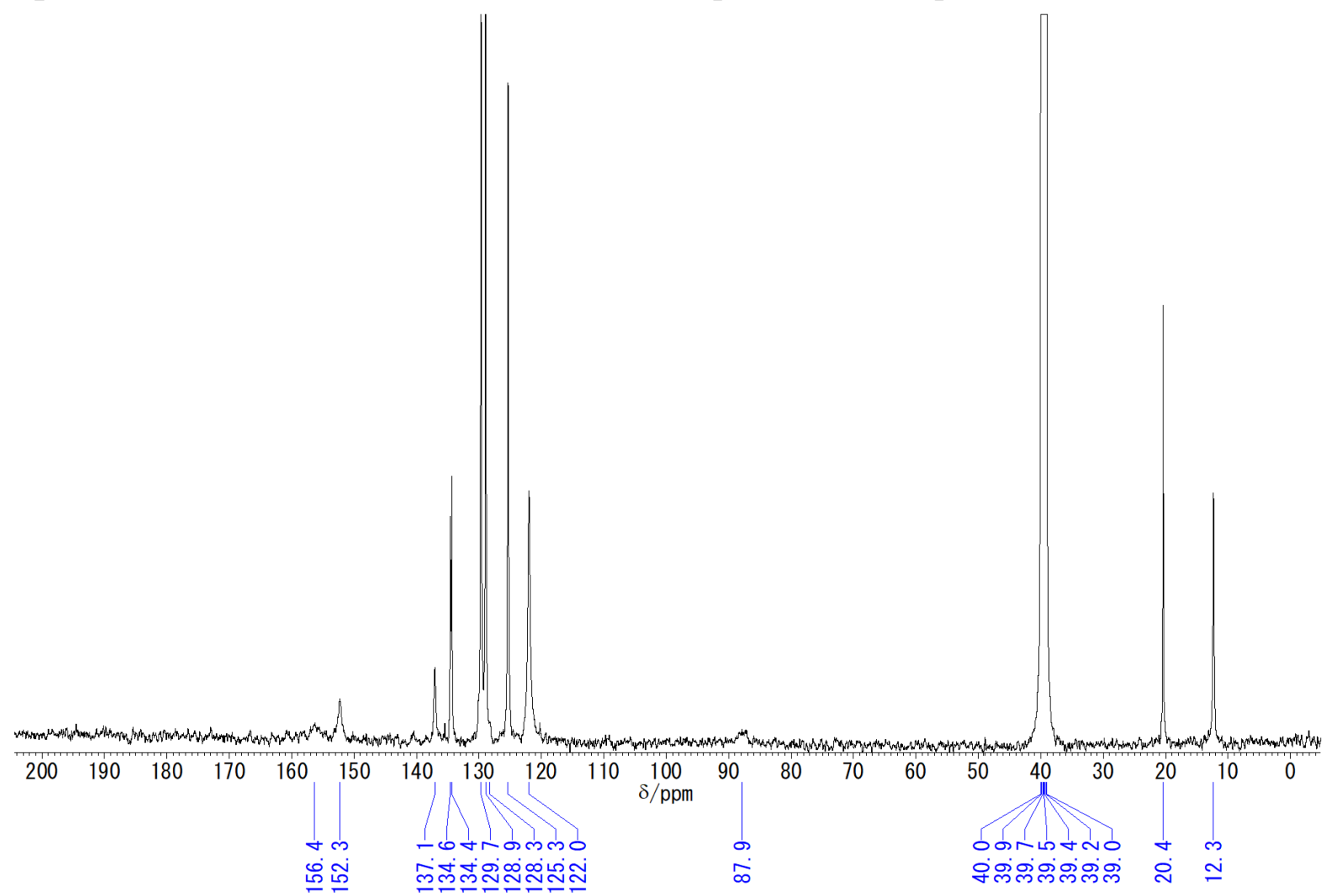

Spectrum S28. ${ }^{13} \mathrm{C}\left\{{ }^{1} \mathrm{H}\right\}$ NMR (DMSO- $d_{6}, 126 \mathrm{MHz}$ ) spectrum of compound 3k. 


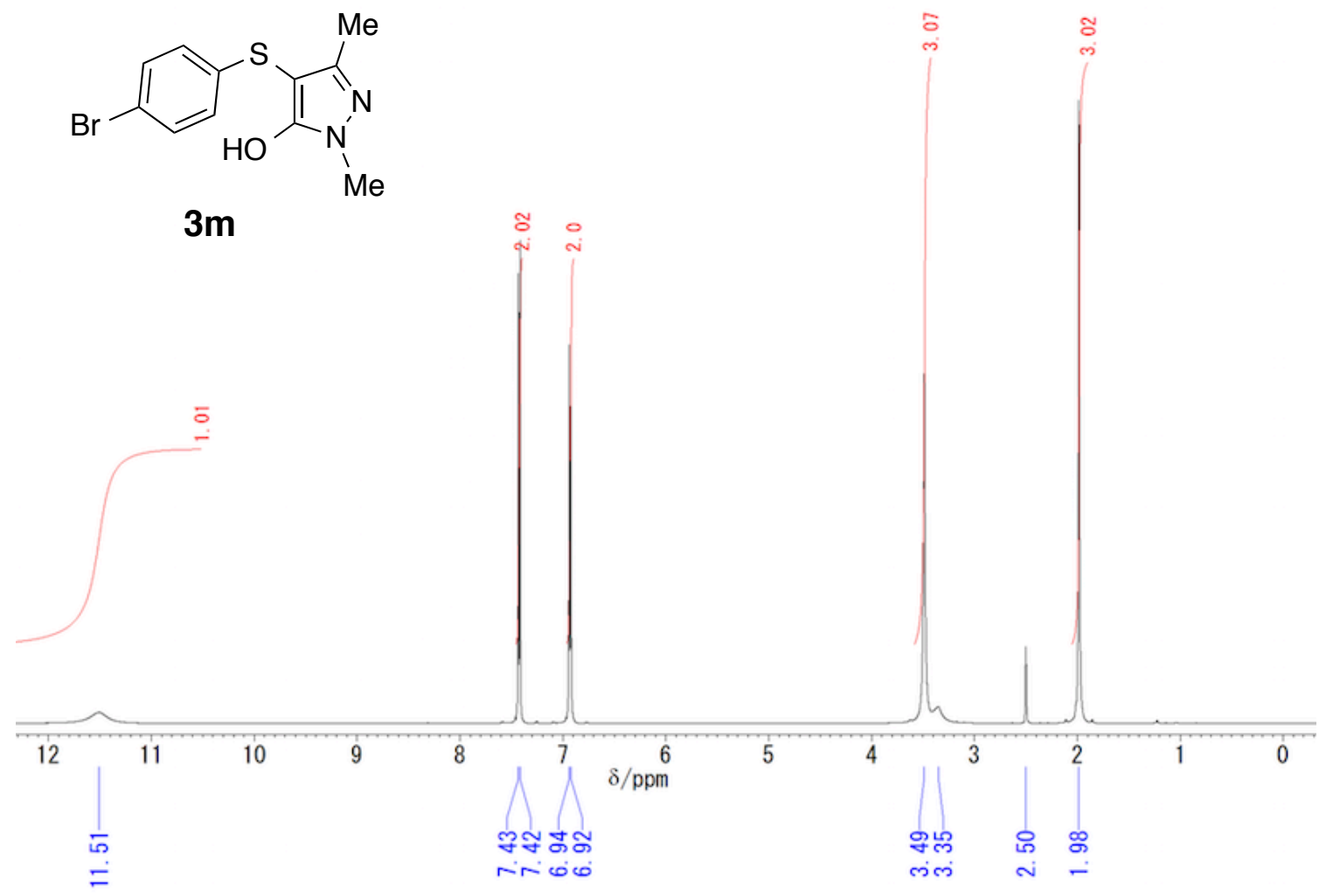

Spectrum S29. ${ }^{1} \mathrm{H}$ NMR (DMSO- $d_{6}, 500 \mathrm{MHz}$ ) spectrum of compound 3m.

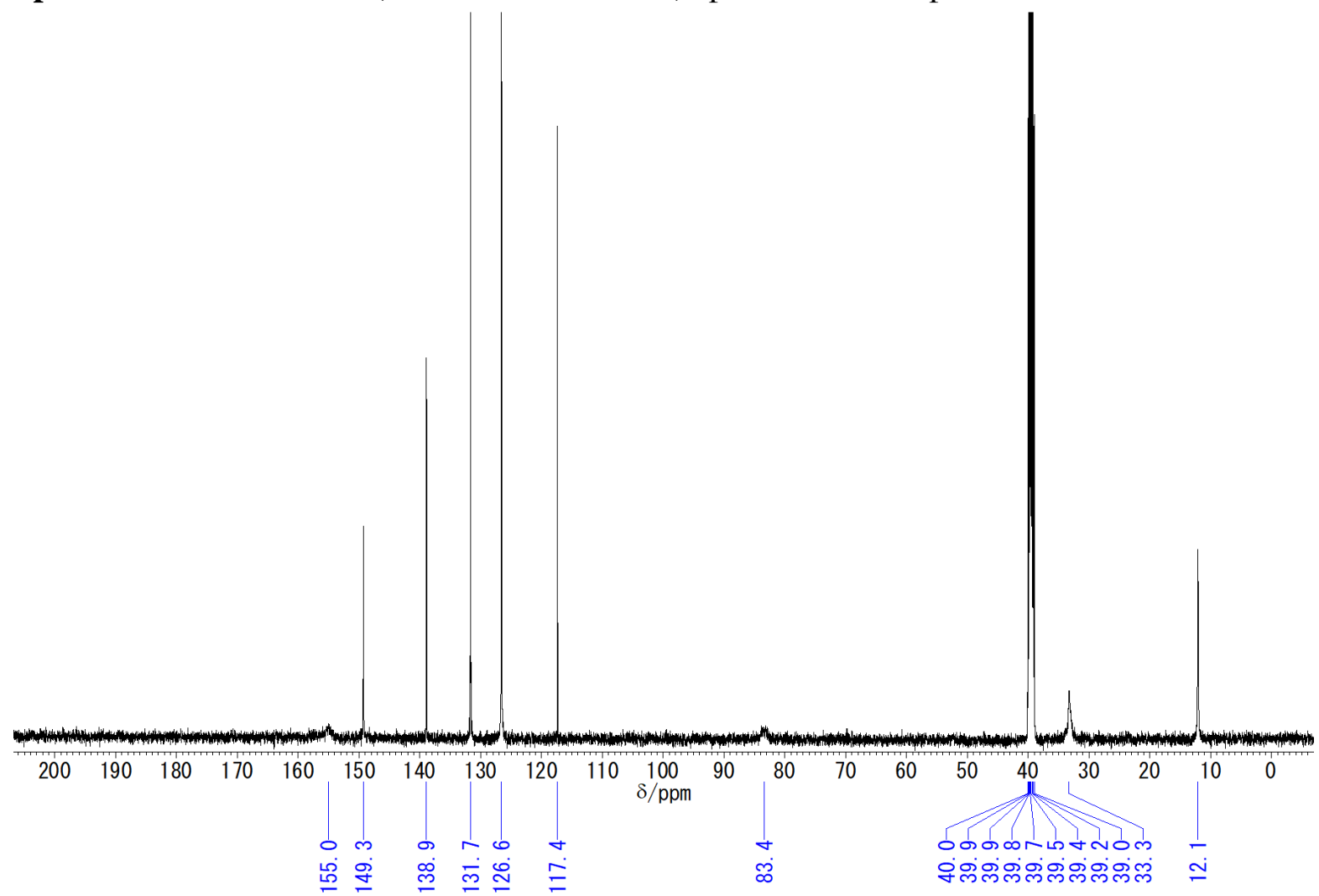

Spectrum S30. ${ }^{13} \mathrm{C}\left\{{ }^{1} \mathrm{H}\right\}$ NMR (DMSO- $d_{6}, 126 \mathrm{MHz}$ ) spectrum of compound 3m. 
<smiles>Cc1ccc(Sc2c(C(C)(C)C)nn(-c3ccccc3)c2O)cc1</smiles>
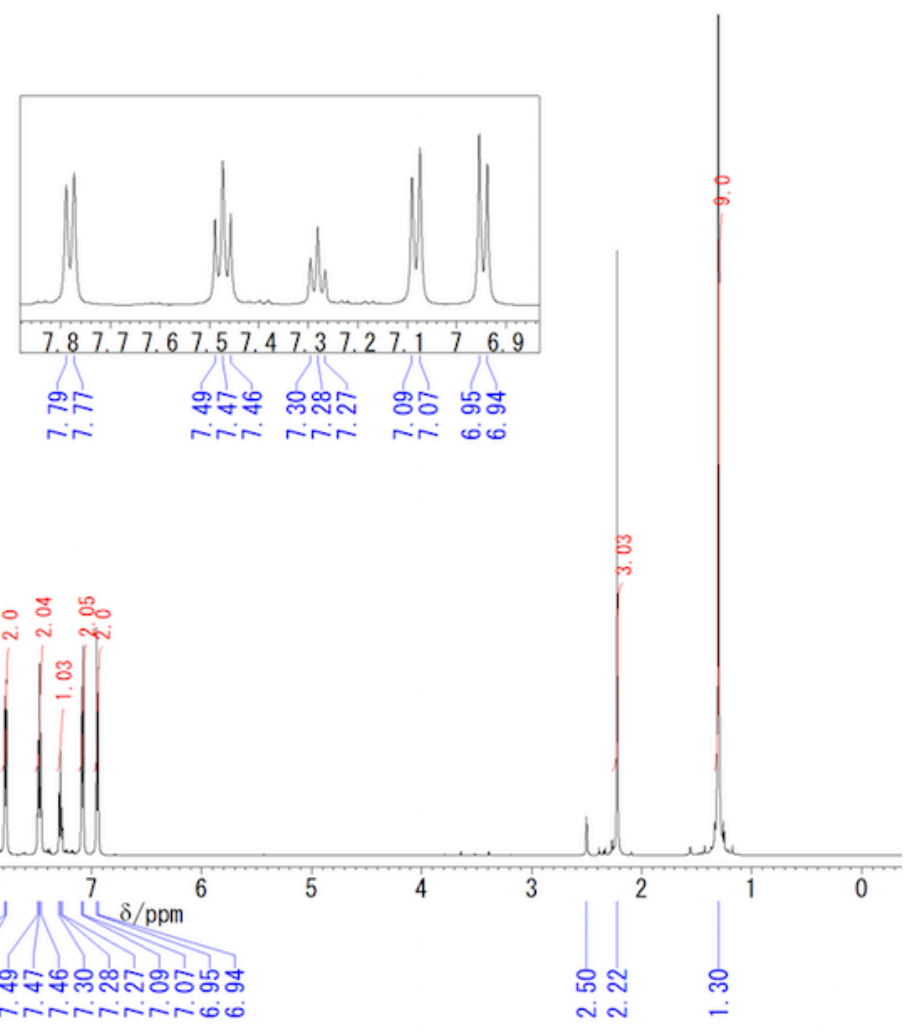

Spectrum S31. ${ }^{1} \mathrm{H}$ NMR (DMSO- $\left.d_{6}, 500 \mathrm{MHz}\right)$ spectrum of compound 3n.

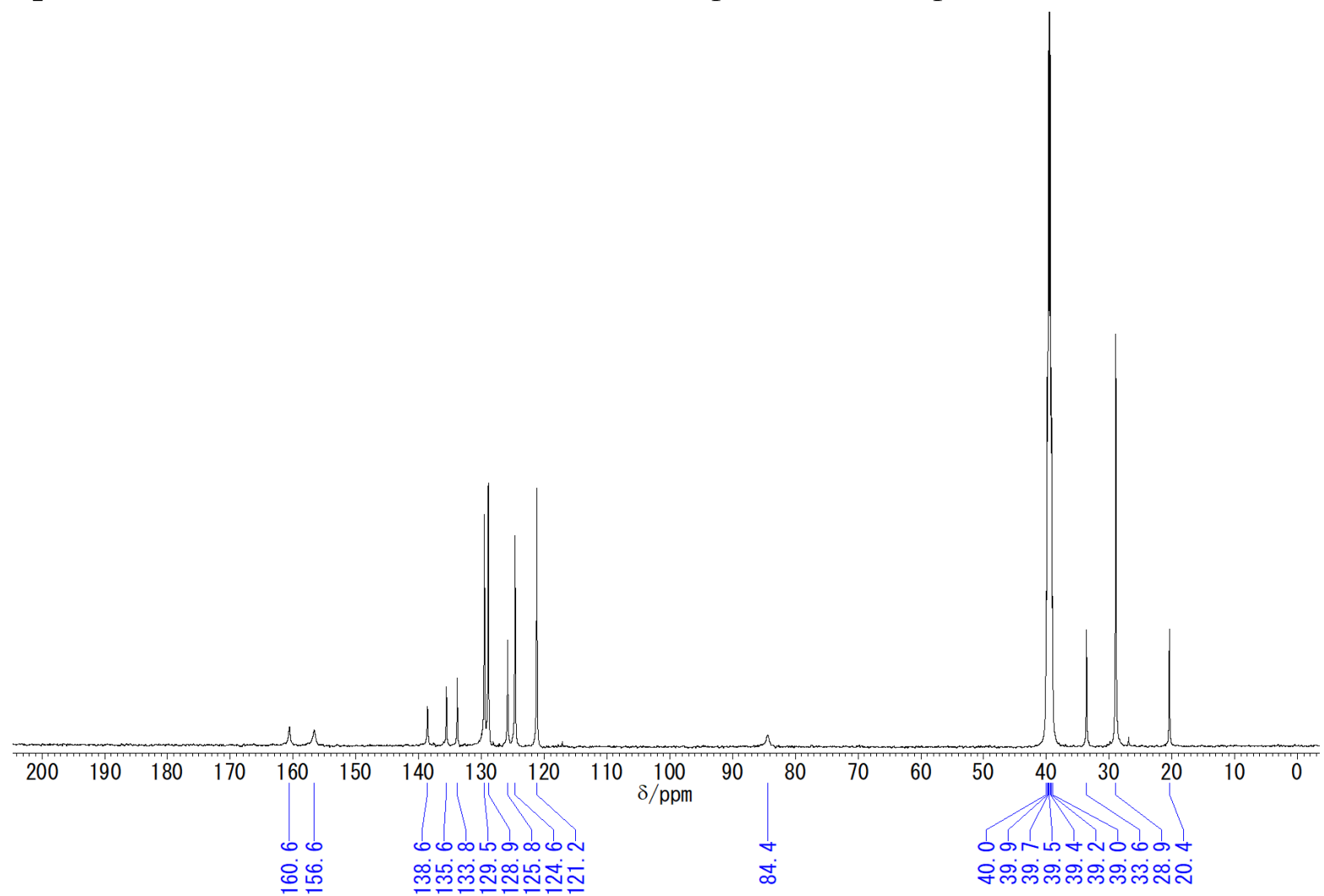

Spectrum S32. ${ }^{13} \mathrm{C}\left\{{ }^{1} \mathrm{H}\right\}$ NMR (DMSO- $d_{6}, 126 \mathrm{MHz}$ ) spectrum of compound 3n. 


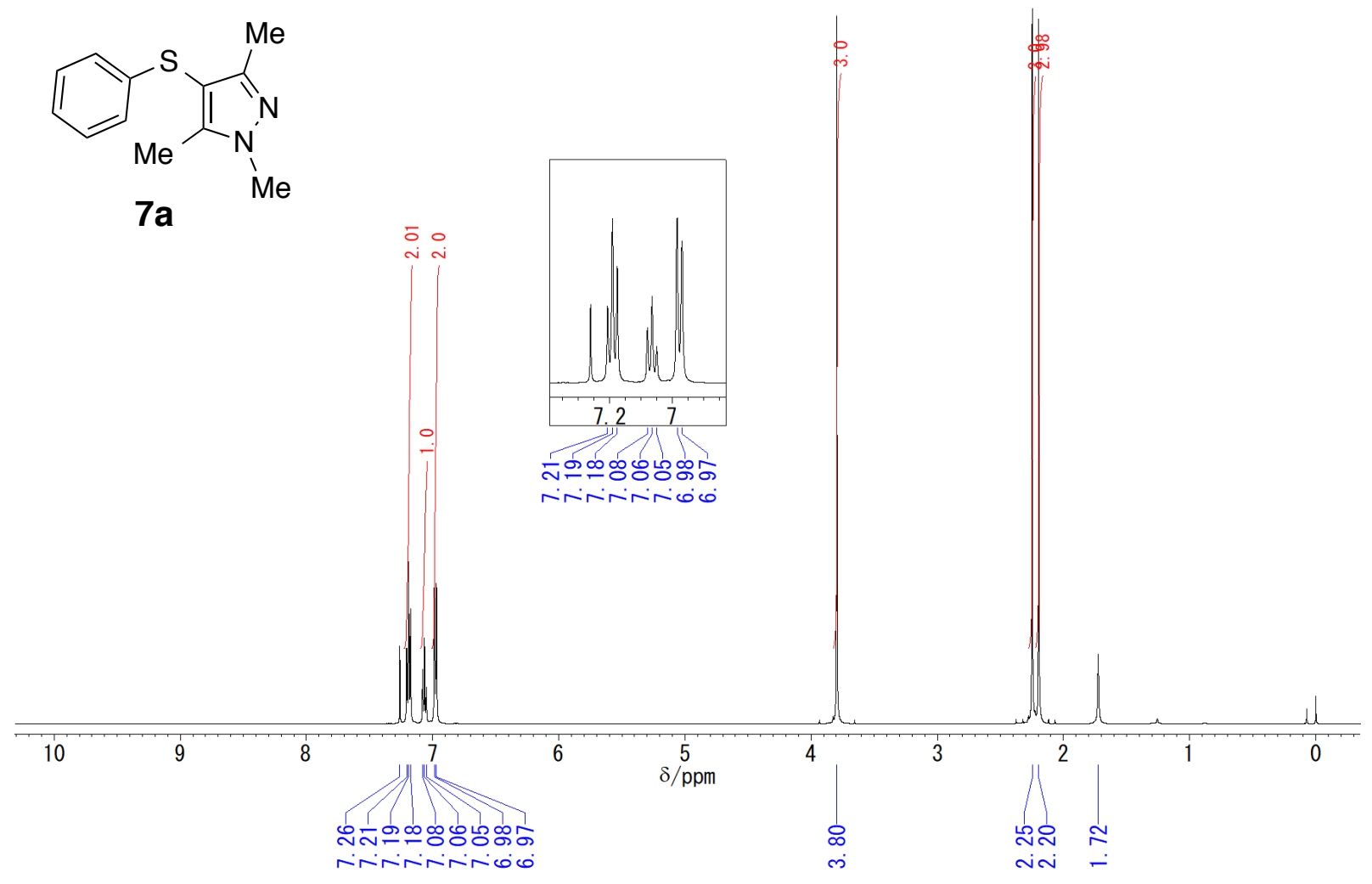

Spectrum S33. ${ }^{1} \mathrm{H}$ NMR $\left(\mathrm{CDCl}_{3}, 500 \mathrm{MHz}\right)$ spectrum of compound 7a.

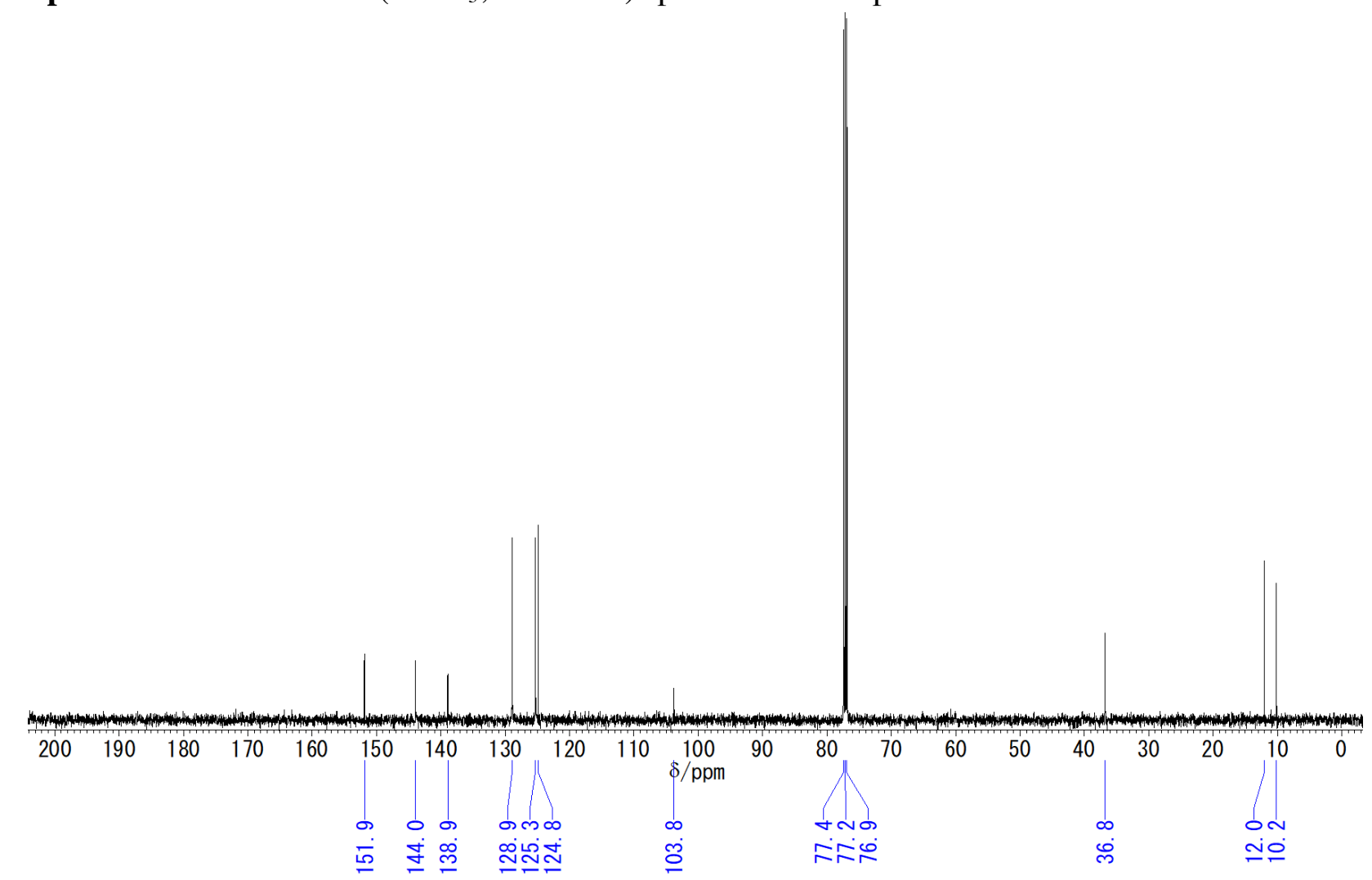

Spectrum S34. ${ }^{13} \mathrm{C}\left\{{ }^{1} \mathrm{H}\right\}$ NMR $\left(\mathrm{CDCl}_{3}, 126 \mathrm{MHz}\right)$ spectrum of compound 7a. 


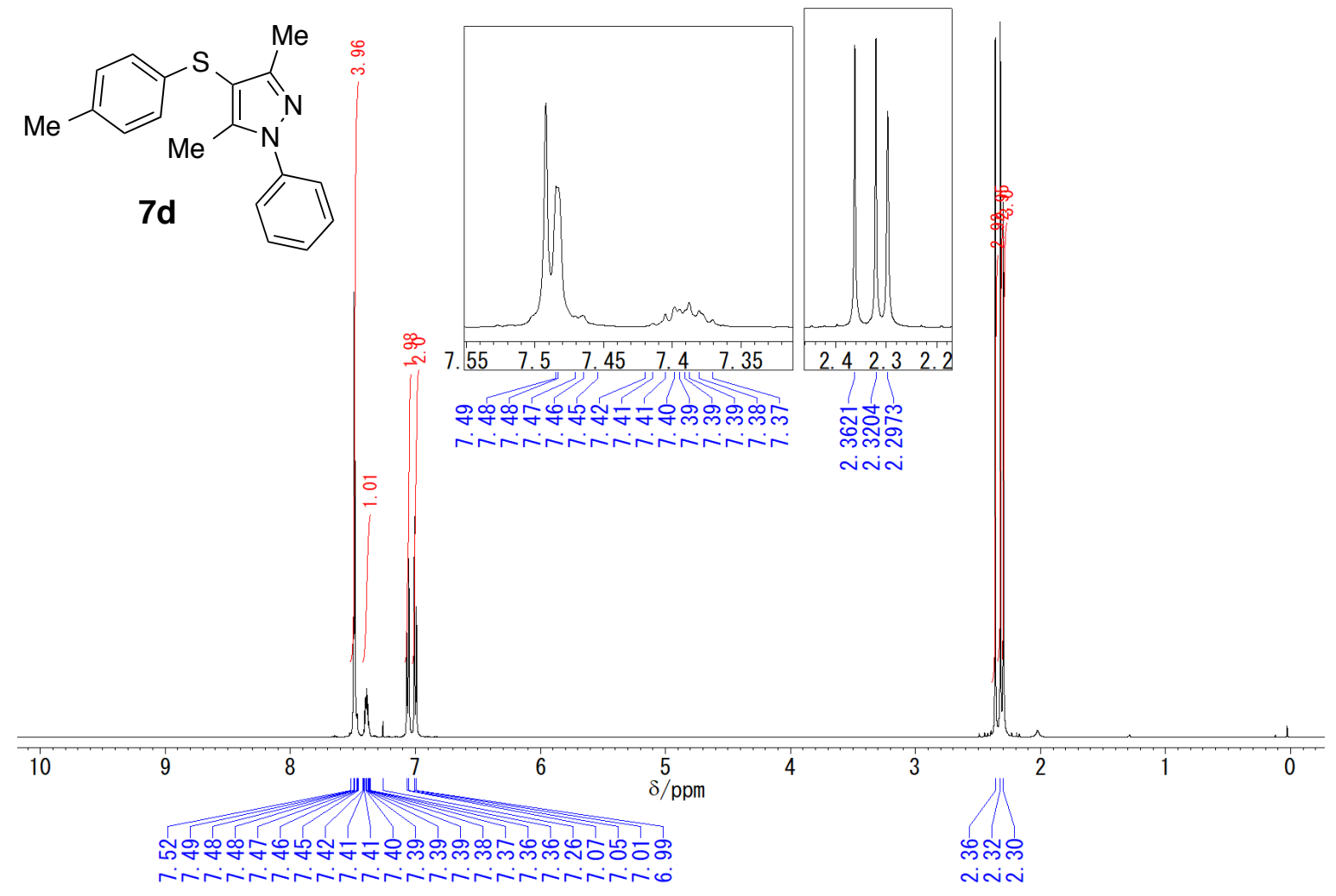

Spectrum S35. ${ }^{1} \mathrm{H}$ NMR $\left(\mathrm{CDCl}_{3}, 500 \mathrm{MHz}\right)$ spectrum of compound 7d.

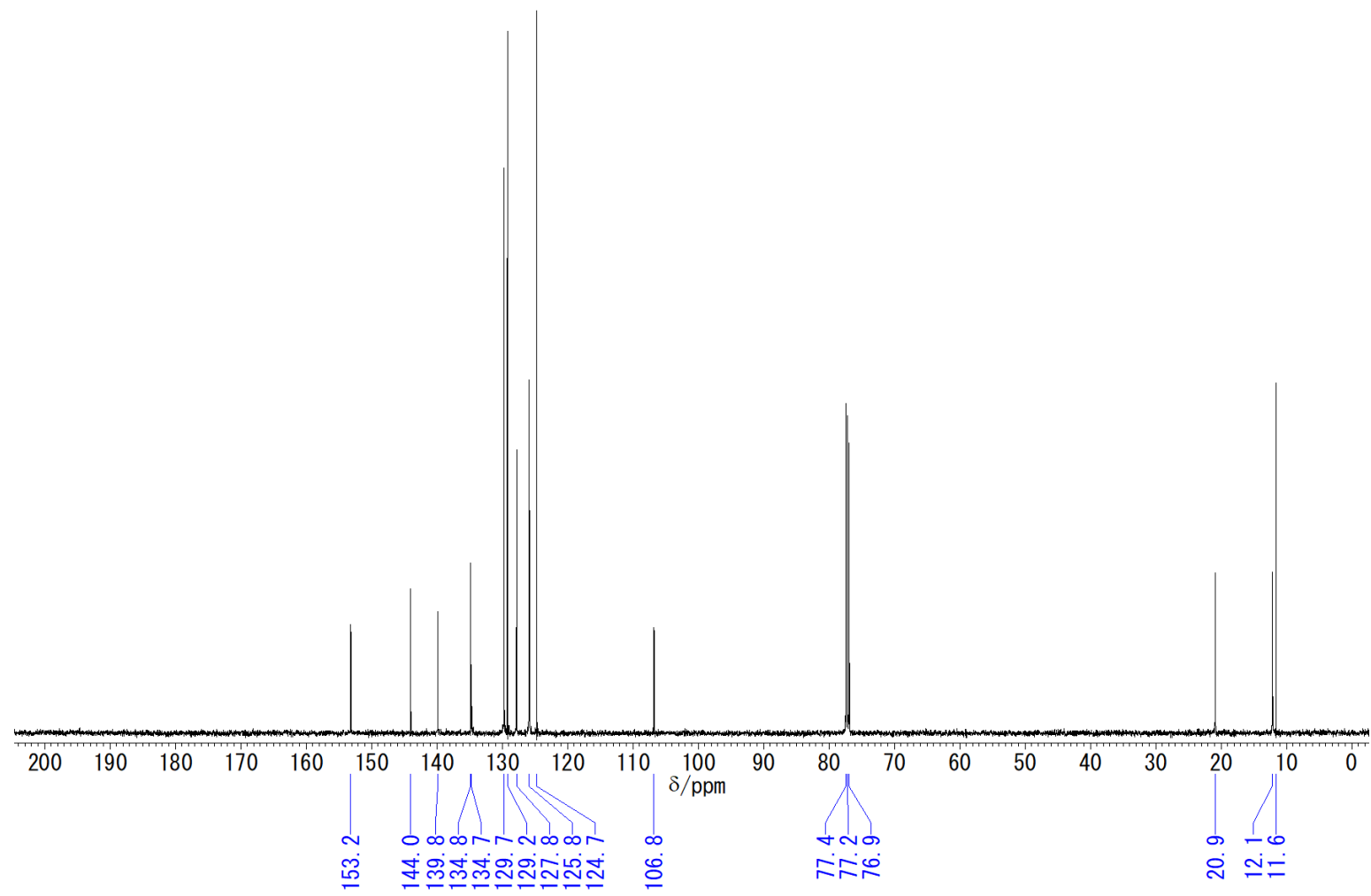

Spectrum S36. ${ }^{13} \mathrm{C}\left\{{ }^{1} \mathrm{H}\right\}$ NMR $\left(\mathrm{CDCl}_{3}, 126 \mathrm{MHz}\right)$ spectrum of compound $7 \mathbf{d}$. 
<smiles>Cc1ccc(Sc2c(C)n[nH]c2C)cc1</smiles>

$7 e$

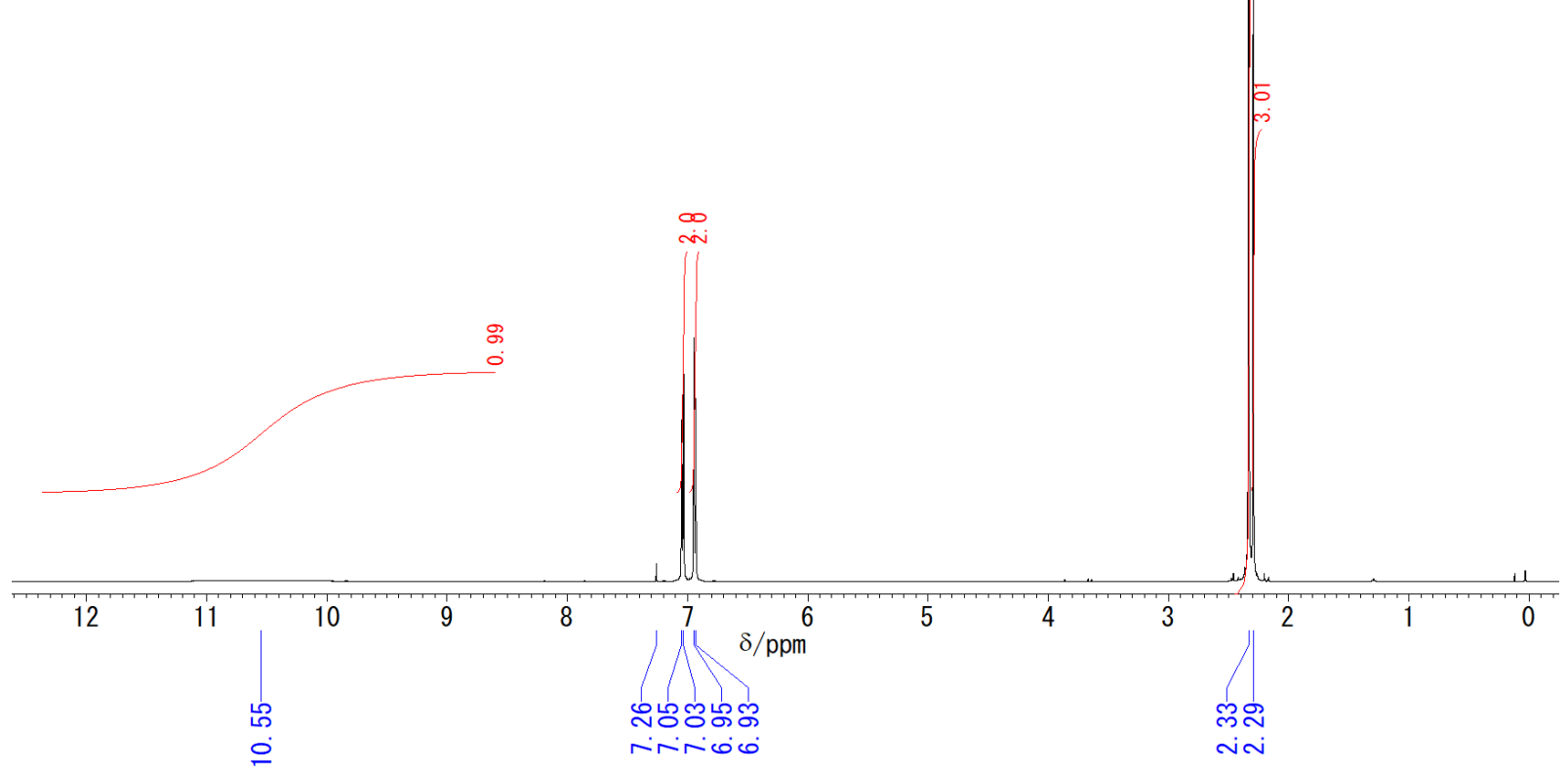

Spectrum S37. ${ }^{1} \mathrm{H}$ NMR $\left(\mathrm{CDCl}_{3}, 500 \mathrm{MHz}\right)$ spectrum of compound 7e.

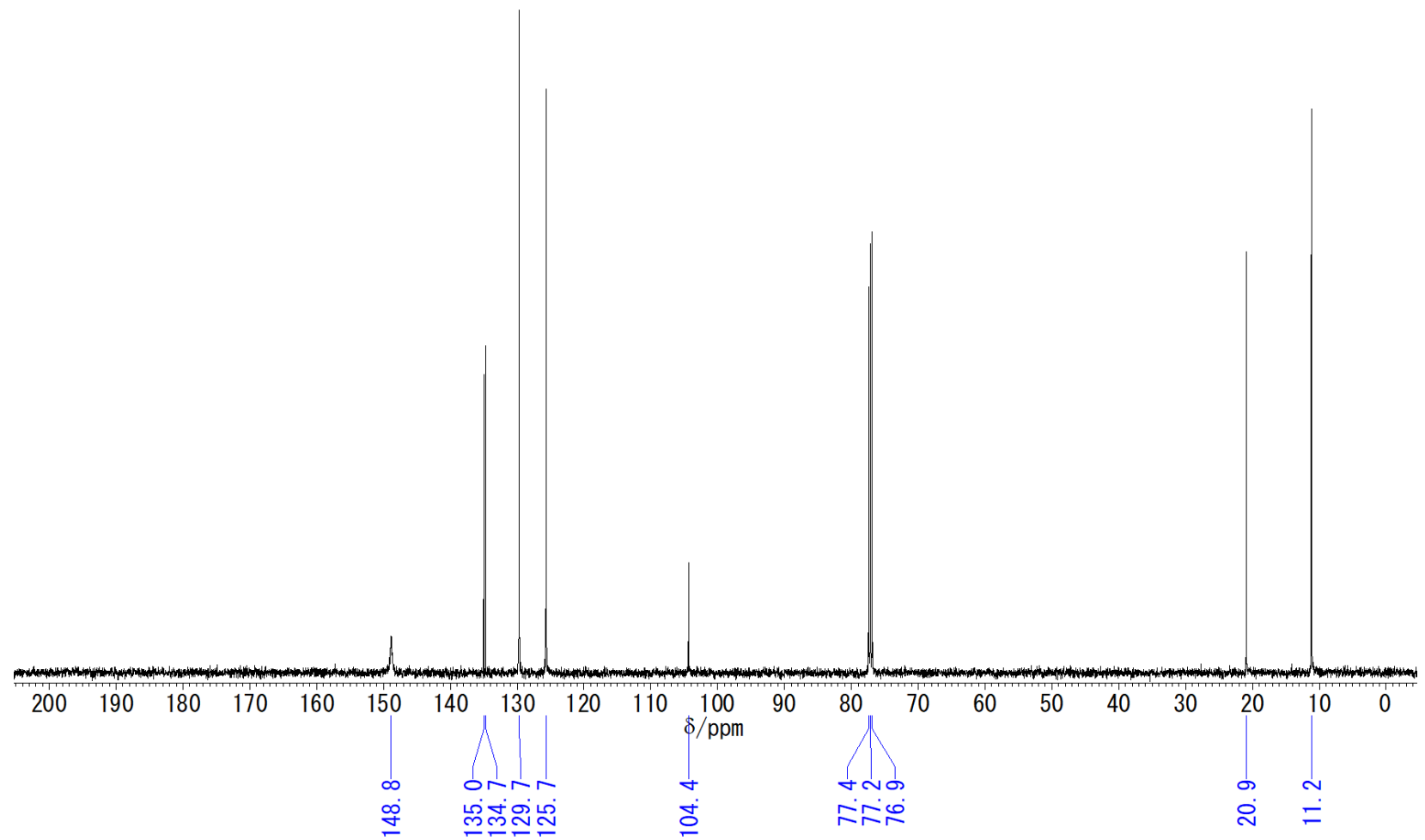

Spectrum S38. ${ }^{13} \mathrm{C}\left\{{ }^{1} \mathrm{H}\right\}$ NMR $\left(\mathrm{CDCl}_{3}, 126 \mathrm{MHz}\right)$ spectrum of compound 7e. 


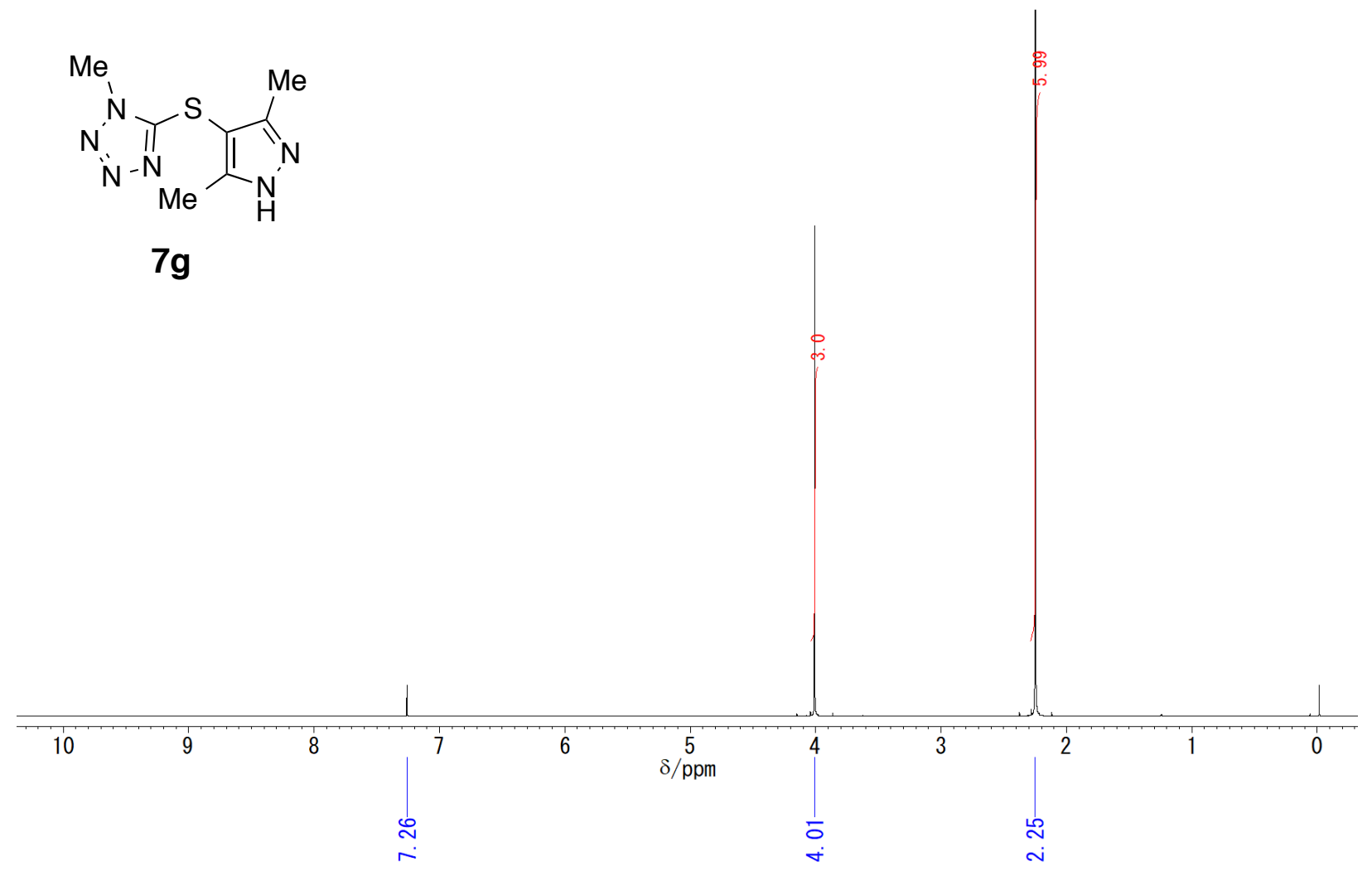

Spectrum S39. ${ }^{1} \mathrm{H}$ NMR $\left(\mathrm{CDCl}_{3}, 500 \mathrm{MHz}\right)$ spectrum of compound $7 \mathrm{~g}$.

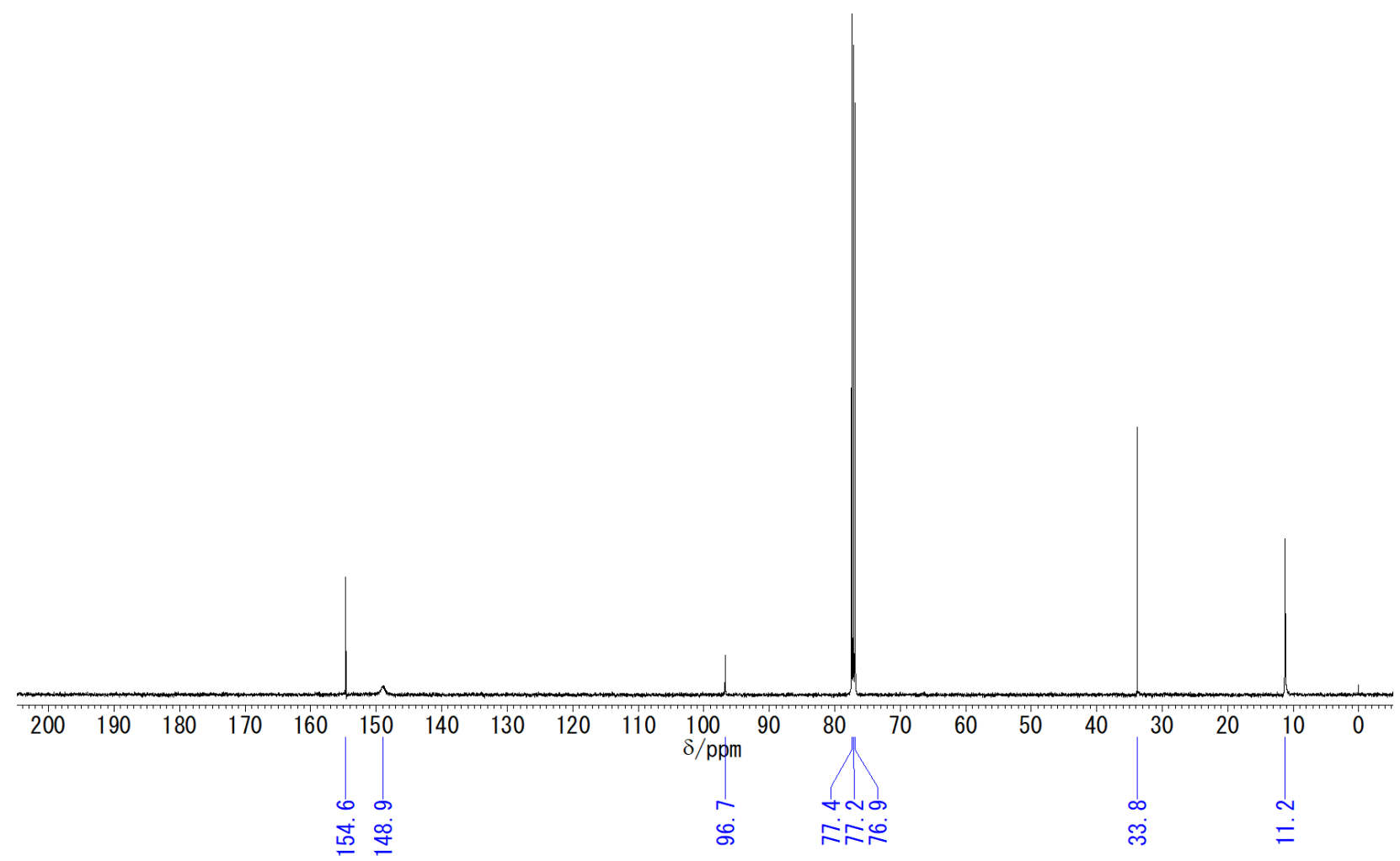

Spectrum S40. ${ }^{13} \mathrm{C}\left\{{ }^{1} \mathrm{H}\right\}$ NMR $\left(\mathrm{CDCl}_{3}, 126 \mathrm{MHz}\right)$ spectrum of compound $7 \mathrm{~g}$. 
<smiles>COc1cc(OC)c(Sc2ccc(C)cc2)c(OC)c1</smiles>

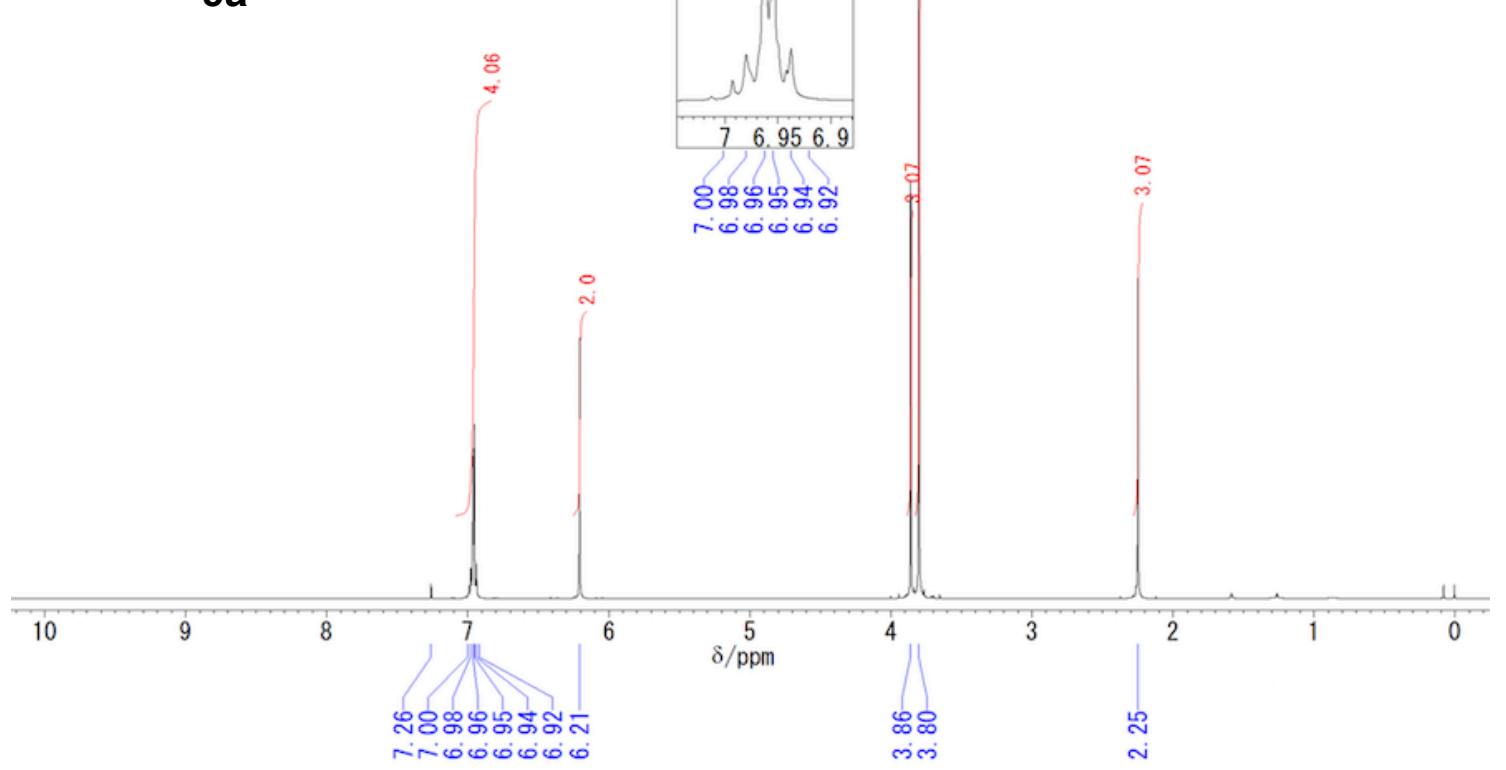

Spectrum S41. ${ }^{1} \mathrm{H}$ NMR $\left(\mathrm{CDCl}_{3}, 500 \mathrm{MHz}\right)$ spectrum of compound 9a.

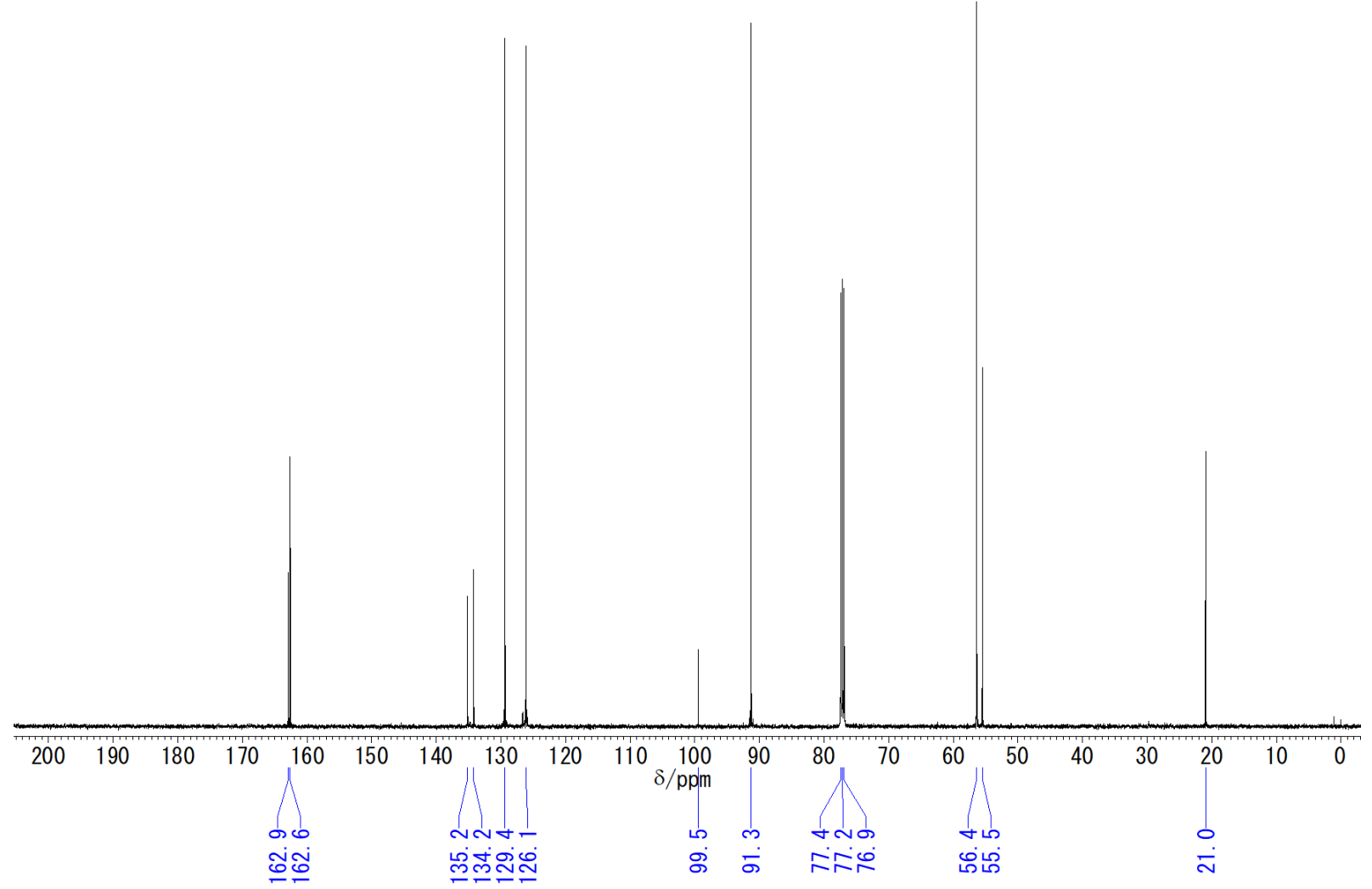

Spectrum S42. ${ }^{13} \mathrm{C}\left\{{ }^{1} \mathrm{H}\right\}$ NMR $\left(\mathrm{CDCl}_{3}, 126 \mathrm{MHz}\right)$ spectrum of compound 9a. 


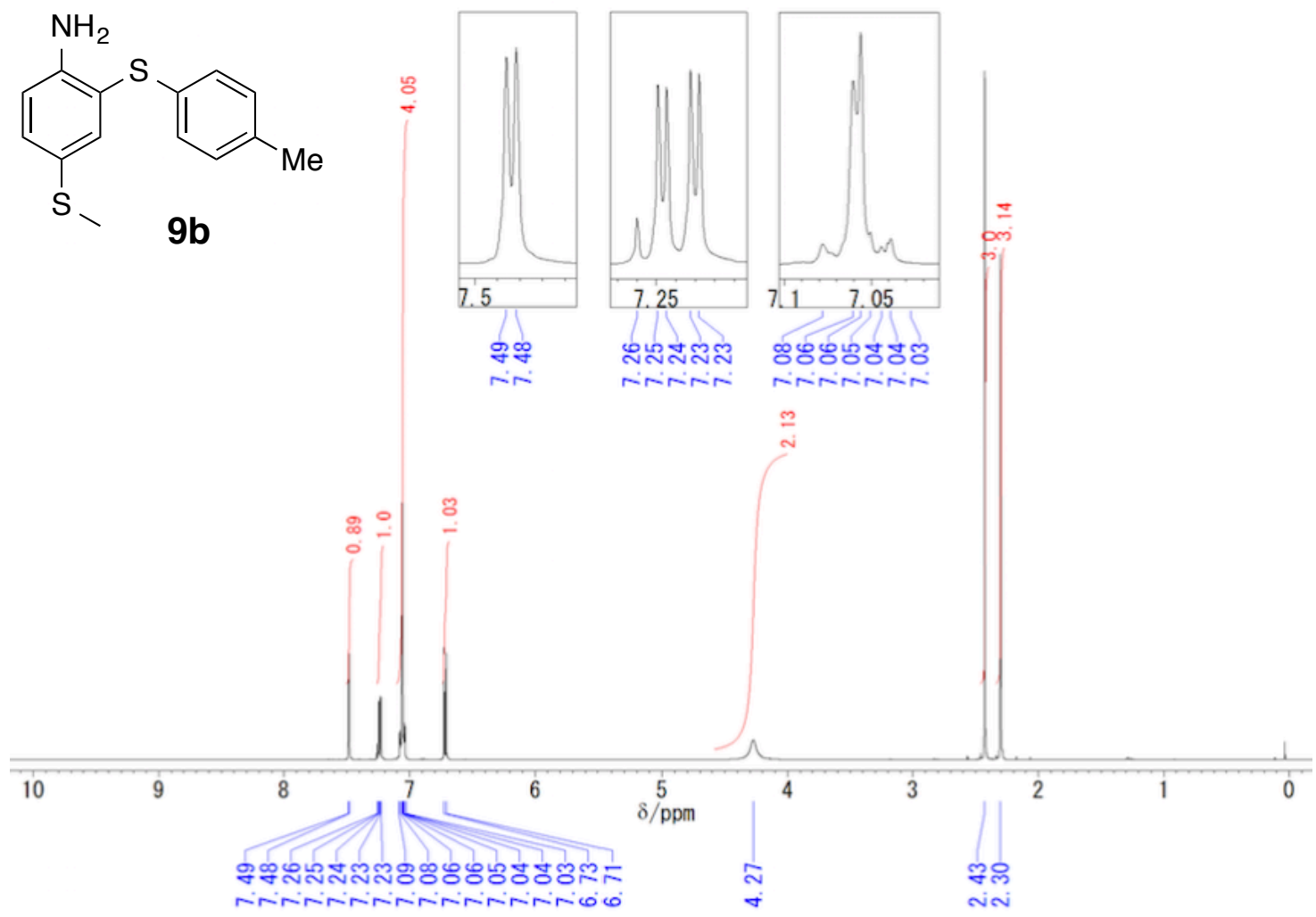

Spectrum S43. ${ }^{1} \mathrm{H}$ NMR $\left(\mathrm{CDCl}_{3}, 500 \mathrm{MHz}\right)$ spectrum of compound $\mathbf{9 b}$.

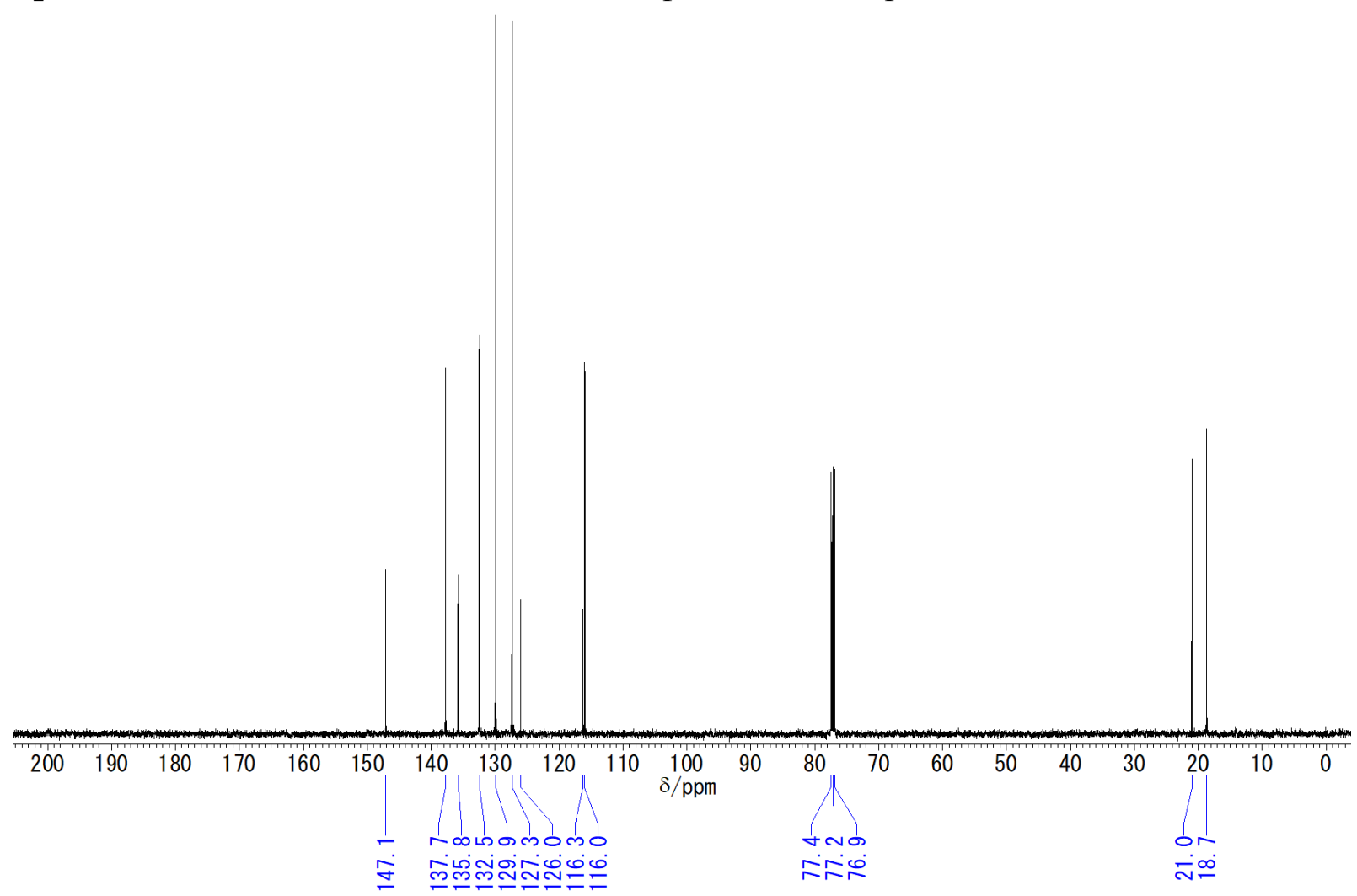

Spectrum S44. ${ }^{13} \mathrm{C}\left\{{ }^{1} \mathrm{H}\right\}$ NMR $\left(\mathrm{CDCl}_{3}, 126 \mathrm{MHz}\right)$ spectrum of compound $\mathbf{9 b}$. 


\section{References}

(S1) Sakai, T.; Kumoi, T.; Ishikawa, T.; Nitta, T.; Iida, H. Comparison of riboflavin-derived flavinium salts applied to catalytic $\mathrm{H}_{2} \mathrm{O}_{2}$ oxidations. Org. Biomol. Chem. 2018, 16, 3999-4007.

(S2) (a) Ménová, P.; Cibulka, R. Insight into the catalytic activity of alloxazinium and isoalloxazinium salts in the oxidations of sulfides and amines with hydrogen peroxide. J. Mol. Catal. A-Chem. 2012, 363-364, 362-370. (b) Cibulka, R. Artificial Flavin Systems for Chemoselective and Stereoselective Oxidations. Eur. J. Org. Chem., 2015, 2015, 915-932.

(S3) König, B.; Pelka, M.; Zieg, H.; Ritter, T.; Bouas-Laurent, H.; Bonneau, R.; Desvergne, J.-P. Photoinduced electron transfer in a phenothiazine-riboflavin dyad assembled by zinc-imide coordination in Water. J.Am. Chem. Soc. 1999, 121, 1681-1687. 